\title{
A COLD NEPTUNE-MASS PLANET OGLE-2007-BLG-368Lb: COLD NEPTUNES ARE COMMON
}

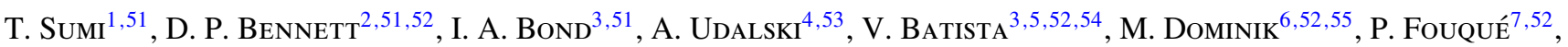

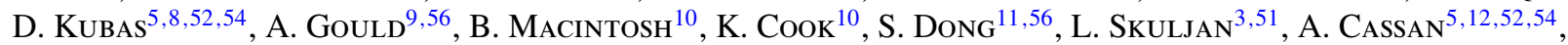 \\ AND
}

F. Abe ${ }^{1}$, C. S. Botzler ${ }^{13}$, A. Fukui ${ }^{1}$, K. Furusawa ${ }^{1}$, J. B. Hearnshaw ${ }^{14}$, Y. Itow ${ }^{1}$, K. Kamiya ${ }^{1}$, P. M. Kilmartin ${ }^{15}$,

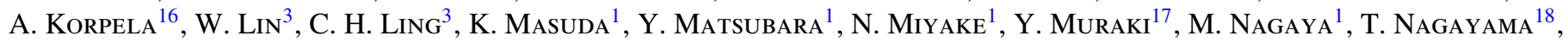
K. Ohnishi ${ }^{19}$, T. Okumura ${ }^{1}$, Y. C. Perrott ${ }^{13}$, N. Rattenbury ${ }^{13}$, To. Saito ${ }^{20}$, T. SAko ${ }^{1}$, D. J. Sullivan ${ }^{16}$, W. L. Sweatman ${ }^{3}$, P. J. Tristram ${ }^{15}$, P. C. M. Yock ${ }^{13}$

(The MOA COllaboration)

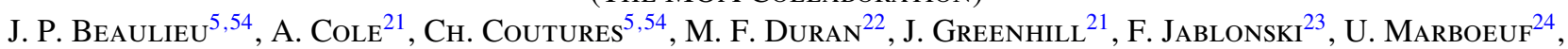
E. Martioli ${ }^{23}$, E. Pedretti ${ }^{6}$, O. Pejchi ${ }^{9}$, P. Rojo ${ }^{22}$, M. D. Albrow ${ }^{14}$, S. Brillant $^{56}$, M. Bode $^{25}$, D. M. Bramich ${ }^{26}$, M. J. Burgdorf ${ }^{27,28}$, J. A. R. Calddwell ${ }^{29}$, H. Calitz ${ }^{30}$, E. Corrales ${ }^{5,54}$, S. Dieters ${ }^{5,21,54}$, D. Dominis Prester ${ }^{31}$,

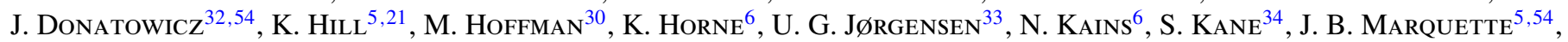
R. Martin ${ }^{35}$, P. Meintues ${ }^{30}$, J. Menzies ${ }^{36}$, K. R. Pollard ${ }^{14}$, K. C. Sahu ${ }^{37}$, C. Snodgrass ${ }^{56}$, I. Steele ${ }^{25}$, R. Street ${ }^{38}$, Y. TSAPRAs ${ }^{38}$, J. WAMBSGANSS $^{12}$, A. Williams ${ }^{35}$, M. ZuB $^{12}$

(The PLANET Collaboration)

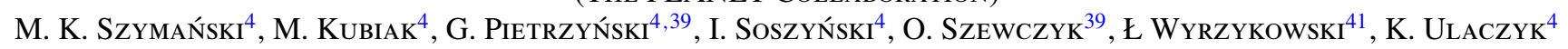
(The OGLE COLlaboration)

W. Allen ${ }^{41}$, G. W. Christie ${ }^{42}$, D. L. DePoy ${ }^{43}$, B. S. Gaudi ${ }^{9}$, C. Han ${ }^{44}$, J. JanCZaK ${ }^{9}$, C.-U. LeE ${ }^{45}$, J. McCormick ${ }^{46}$, F. Mallia ${ }^{47}$, B. Monard ${ }^{48}$, T. Natusch ${ }^{49}$, B.-G. Park ${ }^{45}$, R. W. Pogge ${ }^{9}$ and R. Santallo ${ }^{50}$

(THE $\mu$ FUN COLLABORATION)

${ }^{1}$ Solar-Terrestrial Environment Laboratory, Nagoya University, Nagoya 464-8601, Japan; sumi@ stelab.nagoya-u.ac.jp, abe@ stelab.nagoya-u.ac.jp, afukui@stelab.nagoya-u.ac.jp, furusawa@stelab.nagoya-u.ac.jp, itow@stelab.nagoya-u.ac.jp,kkamiya@ @stelab.nagoya-u.ac.jp, kmasuda@stelab.nagoya-u.ac.jp, ymatsu@ stelab.nagoya-u.ac.jp,nmiyake@ stelab.nagoya-u.ac.jp, mnagaya@stelab.nagoya-u.ac.jp, okumurat@ stelab.nagoya-u.ac.jp, sako@stelab.nagoya-u.ac.jp

2 Department of Physics, University of Notre Dame, Notre Dame, IN 46556, USA; bennett@nd.edu

${ }^{3}$ Institute of Information and Mathematical Sciences, Massey University, Private Bag 102-904, North Shore Mail Centre, Auckland, New Zealand; i.a.bond@massey.ac.nz, 1.skuljan@massey.ac.nz,w.lin@massey.ac.nz, c.h.ling@massey.ac.nz,w.sweatman@massey.ac.nz

${ }^{4}$ Warsaw University Observatory, Al. Ujazdowskie 4, 00-478 Warszawa, Poland; udalski@astrouw.edu.pl, msz@astrouw.edu.pl, mk@astrouw.edu.pl, pietrzyn@astrouw.edu.pl, soszynsk@astrouw.edu.pl, szewczyk@astrouw.edu.pl,kulaczyk@astrouw.edu.pl

${ }^{5}$ Institut d'Astrophysique de Paris, UMR7095 CNRS, Université Pierre \& Marie Curie, 98 bis boulevard Arago, 75014 Paris, France; batista@iap.fr, dkubas@iap.fr, cassan@iap.fr, beaulieu@iap.fr, coutures@iap.fr, marquett@iap.fr

${ }^{6}$ SUPA, Physics \& Astronomy, North Haugh, St Andrews, KY16 9SS, UK; md35@ @t-andrews.ac.uk, kdh1@st-andrews.ac.uk

${ }^{7}$ Laboratoire d'Astrophysique (CNRS), Univ. Paul Sabatier - Toulouse 3, 14, avenue Edouard Belin, F-31400 Toulouse, France; pfouque@ast.obs-mip.fr ${ }^{8}$ European Southern Observatory, Casilla 19001, Vitacura 19, Santiago, Chile

${ }^{9}$ Department of Astronomy, Ohio State University, 140 W. 18th Ave., Columbus, OH 43210, USA; dong@ astronomy.ohio-state.edu, gaudi@astronomy.ohio-state.edu, gould@astronomy.ohio-state.edu, pogge@astronomy.ohio-state.edu

${ }^{10}$ Lawrence Livermore National Laboratory, IGPP, P.O. Box 808, Livermore, CA 94551, USA

${ }^{11}$ Institute for Advanced Study, Princeton, NJ 08540, USA, dong@ias.edu

12 Astronomisches Rechen-Institut, Zentrum für Astronomie, Heidelberg University, Mönchhofstr. 12-14, 69120 Heidelberg, Germany

${ }^{13}$ Department of Physics, University of Auckland, Private Bag 92019, Auckland, New Zealand; c.botzler@auckland.ac.nz, p.yock@auckland.ac.nz, yper006@aucklanduni.ac.nz

${ }^{14}$ University of Canterbury, Department of Physics and Astronomy, Private Bag 4800, Christchurch 8020, New Zealand

${ }_{15}$ Mt. John Observatory, P.O. Box 56, Lake Tekapo 8770, New Zealand

${ }^{16}$ School of Chemical and Physical Sciences, Victoria University, Wellington, New Zealand; a.korpela@ niwa.co.nz, denis.sullivan@vuw.ac.nz

${ }^{17}$ Department of Physics, Konan University, Nishiokamoto 8-9-1, Kobe 658-8501, Japan

${ }^{18}$ Department of Physics and Astrophysics, Faculty of Science, Nagoya University, Nagoya 464-8602, Japan

${ }_{19}$ Nagano National College of Technology, Nagano 381-8550, Japan

${ }^{20}$ Tokyo Metropolitan College of Industrial Technology, Tokyo 116-8523, Japan

${ }^{21}$ University of Tasmania, School of Maths and Physics, Private bag 37, GPO Hobart, Tasmania 7001, Australia

${ }^{22}$ Department of Astronomy, Universidad de Chile, Santiago, Chile

${ }^{23}$ Instituto Nacional de Pesquisas Espaciais, Sao Jose dos Campos, SP, Brazil

${ }^{24}$ Observatoire de Besanon, BP 1615, 25010 Besanon Cedex, France

${ }^{25}$ Astrophysics Research Institute, Liverpool John Moores University, Twelve Quays House, Egerton Wharf, Birkenhead CH41 1LD, UK

${ }^{26}$ European Southern Observatory, Karl-Schwarzschild-Straße 2, 85748 Garching bei München, Germany

${ }^{27}$ Deutsches SOFIA Institut, Universitaet Stuttgart, Pfaffenwaldring 31, 70569 Stuttgart, Germany

28 OFIA Science Center, NASA Ames Research Center, Mail Stop N211-3, Moffett Field, CA 94035, USA; mburgdorf@ sofia.usra.edu

${ }^{29}$ McDonald Observatory, 16120 St Hwy Spur 78 \#2, Fort Davis, TX 79734, USA; caldwell@ astro.as.utexas.edu

${ }^{30}$ Department of Physics/Boyden Observatory, University of the Free State, Bloemfontein 9300, South Africa; calitzjj.sci@mail.uovs.ac.za

${ }^{31}$ Physics Department, Faculty of Arts and Sciences, University of Rijeka, 51000 Rijeka, Croatia

32 Technical University of Vienna, Department of Computing, Wiedner Hauptstrasse 10, Vienna, Austria

${ }^{33}$ Niels Bohr Institute, Astronomical Observatory, Juliane Maries Vej 30, DK-2100 Copenhagen, Denmark

${ }^{34}$ NASA Exoplanet Science Institute, Caltech, MS 100-22, 770 South Wilson Avenue, Pasadena, CA 91125, USA

${ }^{35}$ Perth Observatory, Walnut Road, Bickley, Perth 6076, Australia; rmartin@ physics.uwa.edu.au, andrew@ physics.uwa.edu.au

${ }^{36}$ South African Astronomical Observatory, P.O. Box 9 Observatory 7935, South Africa

${ }^{37}$ Space Telescope Science Institute, 3700 San Martin Drive, Baltimore, MD 21218, USA; ksahu@ stsci.edu

${ }^{38}$ Las Cumbres Observatory Global Telescope Network, 6740B Cortona Dr, Suite 102, Goleta, CA 93117, USA; rstreet@ lcogt.net, ytsapras@1cogt.net

${ }^{39}$ Universidad de Concepción, Departamento de Fisica, Casilla 160-C, Concepción, Chile 


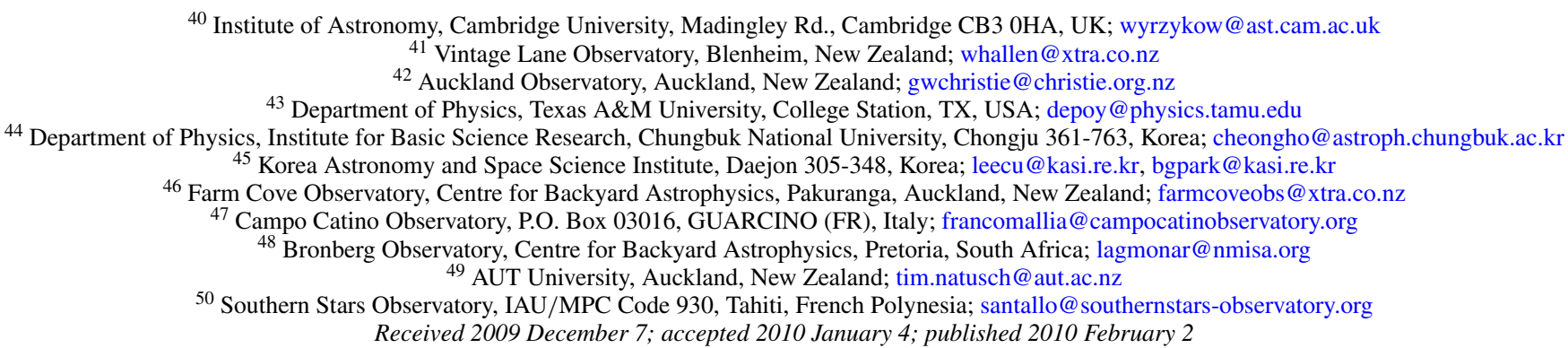

ABSTRACT

We present the discovery of a Neptune-mass planet OGLE-2007-BLG-368Lb with a planet-star mass ratio of $q=[9.5 \pm 2.1] \times 10^{-5}$ via gravitational microlensing. The planetary deviation was detected in real-time thanks to the high cadence of the Microlensing Observations in Astrophysics survey, real-time light-curve monitoring and intensive follow-up observations. A Bayesian analysis returns the stellar mass and distance at $M_{l}=0.64_{-0.26}^{+0.21} M_{\odot}$ and $D_{l}=5.9_{-1.4}^{+0.9} \mathrm{kpc}$, respectively, so the mass and separation of the planet are $M_{p}=20_{-8}^{+7} M_{\oplus}$ and $a=3.3_{-0.8}^{+1.4} \mathrm{AU}$, respectively. This discovery adds another cold Neptune-mass planet to the planetary sample discovered by microlensing, which now comprises four cold Neptune/super-Earths, five gas giant planets, and another subSaturn mass planet whose nature is unclear. The discovery of these 10 cold exoplanets by the microlensing method implies that the mass ratio function of cold exoplanets scales as $d N_{\mathrm{pl}} / d \log q \propto q^{-0.7 \pm 0.2}$ with a $95 \%$ confidence level upper limit of $n<-0.35$ (where $d N_{\mathrm{pl}} / d \log q \propto q^{n}$ ). As microlensing is most sensitive to planets beyond the snow-line, this implies that Neptune-mass planets are at least three times more common than Jupiters in this region at the $95 \%$ confidence level.

Key words: gravitational lensing: micro - planetary systems

Online-only material: color figure

\section{INTRODUCTION}

Since the first discovery of exoplanets orbiting main-sequence stars in 1995 (Mayor \& Queloz 1995; Marcy et al. 2005), more than 300 exoplanets have been discovered via the radial velocity method (Mayor et al. 2004) and more than 50 have been detected via their transits (Udalski et al. 2004; Konacki et al. 2005). Several planetary candidates have also been detected via direct imaging (Marois et al. 2008; Lagrange et al. 2009), and astrometry (Pravdo \& Shaklan 2009). Here, we report the tenth exoplanet discovery by the microlensing method, which is another example of a cold, Neptune-mass planet discovered. Although the radial velocity and transit discoveries are more numerous, microlensing is uniquely sensitive to these cold Neptunes, and the microlensing results to date indicate that this class of planets may be the most common type of exoplanet yet discovered.

Liebes (1964) and Mao \& Paczyński (1991) first proposed exoplanet searches via gravitational microlensing. The planet's gravity induces small caustics, which can generate small deviations in standard (Paczyński 1986) single-lens microlensing light curves. Compared to other techniques, microlensing is sensitive to smaller planets, down to an Earth mass (Bennett \& Rhie 1996), and in wider orbits of 1-6 AU. Because microlensing observability does not depend on the light from the lens host star, it is sensitive to planets orbiting faint host stars like M-dwarfs and even brown dwarfs. Furthermore, it is sensitive to distant

\footnotetext{
${ }^{51}$ Microlensing Observations in Astrophysics (MOA).

${ }^{52}$ Probing Lensing Anomalies NETwork (PLANET).

${ }^{53}$ Optical Gravitational Lens Experiment (OGLE).

${ }^{54}$ HOLMES Collaboration.

${ }^{55}$ Royal Society University Research Fellow.

${ }^{56}$ Microlensing Follow Up Network ( $\mu$ FUN).
}

host stars at several kpc from the Sun, which allows the Galactic distribution of planetary systems to be studied.

In 2003, the gravitational microlensing method yielded its first definitive exoplanet discovery (Bond et al. 2004). So far eight planetary systems with nine planets have been found by this technique (Udalski et al. 2005; Beaulieu et al. 2006; Gould et al. 2006; Gaudi et al. 2008; Bennett et al. 2008; Dong et al. 2009b; Janczak et al. 2010), which have very distinct properties from those detected by other techniques. Beaulieu et al. (2006) found a $\sim 5.5$ Earth-mass planet, which was the lowest-mass planet detected at that time. This detection and the discovery of a slightly more massive planet by Gould et al. (2006) demonstrated that microlensing is well suited to detecting low-mass planets at orbital distances that are currently beyond the reach of other methods. At the time of the discovery of these two cold Neptune-mass planets (hereafter "Neptunes") or "super Earths," two Jovian planets had also been found. These discoveries indicate that cold Neptune in orbits beyond the "snow-line" (Ida \& Lin 2004; Laughlin et al. 2004; Kennedy et al. 2006) around late-type stars, are significantly more common than gas giants with frequency of $\geqslant 16 \%$ at $90 \%$ confidence (Gould et al. 2006), which is consistent with theoretical simulations (Ida \& Lin 2004) based on the core accretion model. On the other hand, microlensing has also revealed the most massive M-dwarf planetary companion (Udalski et al. 2005; Dong et al. 2009a), which would likely be difficult to form by core accretion (Laughlin et al. 2004). Gaudi et al. (2008) discovered a system with a Jupiter and a Saturn orbiting an M dwarf in a configuration very similar to that of our solar system. Remarkably, this event yielded a direct measurement of the masses of the planets and the host star that was confirmed by direct observation of the host star. This system (OGLE-2006-BLG-109Lb,c) is the only known multi-planet system with measured masses for the star and planets (aside 
from our own solar system). The light curve of this event also yielded information about the orbit of the Saturn-mass planet which confirms that this system is similar to ours (Bennett et al. 2009). A planet was also found to orbit a very low mass host star or brown dwarf (Bennett et al. 2008), and this planet was also the lowest-mass exoplanet known at the time of its discovery.

Here we report the discovery of another Neptune-mass exoplanet in the microlensing event OGLE-2007-BLG-368. We describe the data sets in Section 2. The light-curve modeling and uncertainty of the parameters are presented in Section 3, and the physical characterization of the lens system is considered in Section 7. In Section 8, we discuss the implications of microlensing planet discoveries for the exoplanet mass function. The discussion and conclusions are given in Section 9.

\section{OBSERVATIONS}

The Optical Gravitational Lensing Experiment (OGLE; Udalski 2003) and Microlensing Observations in Astrophysics (MOA; Bond et al. 2001; Sumi et al. 2003) are conducting microlensing surveys toward the Galactic bulge to find exoplanets. From 2002 to 2008, the OGLE-III survey discovered about 600 microlensing events every year by using $1.3 \mathrm{~m}$ Warsaw telescope with a $0.34 \mathrm{deg}^{2}$ field-of-view (FOV) mosaic CCD camera with its Early Warning System (EWS; Udalski 2003). The data have been analyzed in real time and all kind of deviations from the usual single-lens light curves, including planetary anomalies, have been detected by the EWS (Udalski 2003). The second phase of MOA, MOA-II, carries out a very high cadence photometric survey of the Galactic bulge with the $1.8 \mathrm{~m}$ MOA-II telescope with a $2.2 \mathrm{deg}^{2}$ FOV CCD camera. In $2007,4.5 \mathrm{deg}^{2}$ of the central Galactic bulge were observed every 10 minutes, and additional $45 \mathrm{deg}^{2}$ were observed with a 50 minute cadence. This strategy enables the detection in realtime of planetary deviations in any of the $\sim 500$ microlensing events seen by MOA every year. (Starting in 2010, the new $1.4 \mathrm{deg}^{2}$ OGLE-IV camera will enable OGLE to follow a similar strategy of high-cadence monitoring for planetary signals.)

The microlensing event OGLE-2007-BLG-368 was discovered at (R.A., decl. $)(2000)=(17: 56: 25.96,-32: 14: 14.7)$ $[(l, b)=(358.3,-3 \circ 7)]$ and alerted by the OGLE EWS system (Udalski 2003) on 2007 July 10, and independently detected by MOA and alerted as MOA-2007-BLG-308 on 2007 July 12.

Around UT 12:00 July 20 (JD = 2454302), MOA observed a series of nine points that are all below the point lens light curve, and these are confirmed by a single OGLE point, with higher precision. See Figure 1. The prompt informative data release to the scientific community allowed the SIGNALMEN anomaly detector (Dominik et al. 2007) (now an integral part of the Automated Robotic Terrestrial Exoplanet Microlensing Search (ARTEMiS) system; Dominik et al. 2008) to detect a light-curve anomaly that was passed on to 1-3 members of each of the major microlensing collaborations, such as PLANET, $\mu$ FUN, RoboNet, OGLE, and MOA at UT 19:32 20 July (JD $=2454302.314$ ), that this was a possible planetary anomaly. Given the intensity of microlensing decision-making and the incompleteness of the information flow, this distribution proved only partially adequate and failed to reach the MOA internal alert system. Based on this alert, the $\mu$ FUN SMARTS (CTIO) telescope began obtaining data just $5 \mathrm{hr}$ later, shortly after dusk in Chile, after which the PLANET Danish (La Silla, Chile) and Canopus (Tasmania, Australia) telescopes also began observations. Although the MOA observer did not receive this alert, its high-cadence survey enabled good coverage of a steep rise due to the caustic entrance in the light curve the next night, which triggered MOA's real-time anomaly alert system to circulate an alert, calling for the firm detection of the anomaly, based on its own data at UT 15:58 July 21 $(\mathrm{JD}=2454303.16528)$. Here the real-time anomaly alert system adds new data points on the light curves within 5 minutes after exposures to search for deviations from the single-lens light curve. This continuous early coverage proved crucial for the interpretation of the planetary anomaly. See Figure 1. Prompted by these anomaly alerts, MOA-II, OGLE-III and other telescopes from PLANET and $\mu$ FUN began intensive follow-up observations, which densely covered the second peak, due to the caustic exit, and less densely for about 50 days.

Twelve light curves were collected by seven telescopes. MOA-II 1.8 m (Mt. John, New Zealand) obtained 1577 images in the MOA-Red wide band, which corresponds roughly to a combined $I+R$ filter. OGLE-III $1.3 \mathrm{~m}$ (Las Campanas, Chile) obtained 12 images in the $V$ band and 733 in $I$. $\mu$ FUN SMARTS $1.3 \mathrm{~m}$ (CTIO, Chile) obtained 22 images in $V, 137$ in $I$, and 128 in $H$. PLANET SAAO $1 \mathrm{~m}$ (SAAO, South Africa) obtained 9 images in $V$ and 60 in $I$. PLANET Canopus $1 \mathrm{~m}$ (Tasmania) obtained 50 images in $I$. PLANET Danish $1.54 \mathrm{~m}$ (La Silla) obtained 20 images in $V$ and 129 in $I$. PLANET OPD/LNA 0.6 m (Brazil) obtained 122 unfiltered images.

The photometry of this event was much more difficult than the photometry of most microlensing events due to a much brighter star located approximately $1^{\prime \prime} .1$ to the NW of the source star. This caused very severe problems with standard pointspread function (PSF)-fitting photometry approaches, such as DOPHOT (Schechter et al. 1993), so the only viable approach was the difference image analysis (DIA) method (Tomany \& Crotts 1996; Alard \& Lupton 1998; Alard 2000). The images were reduced by three different implementations of DIA photometry. OGLE $V$ and $I$ and CTIO $I$ images were reduced by the standard OGLE DIA pipeline (Udalski 2003). Other images were reduced by both the MOA DIA pipeline (Bond et al. 2001) and a version of pySIS (ver. 3.0; Albrow et al. 2009), partly based on ISIS (Alard \& Lupton 1998), but using a numerical kernel (Bramich 2008). In the MOA DIA pipeline, PSF photometry was performed on the difference images with various reference images and PSF fitting radii.

The resulting MOA DIA light curves, the pySIS light curves, and OGLE DIA light curves were compared and the best one was selected in each data set as follows. First, the planetary deviation at HJD-245000 = 4300-4305 was removed from each light curve, and these planet-free light curves were fitted with a single-lens model with xallarap (binary orbital motion of the source). Details are discussed in Section 3. The photometric reduction yielding the smallest variance from the best model in these planet-free fits was selected to use for further analysis. For each data set, the error bars were rescaled so that $\chi^{2} /($ dof $) \approx 1$ in the planet-free single-lens fit. For CTIO $V$ and $H$ which have very few data points unaffected by the planetary deviation, this same procedure was followed including the planetary deviation using the best fit planetary model to all the data sets.

Figure 1 shows the light curves of this event around the peak and the planetary deviation.

\section{LIGHT-CURVE MODELING}

The negative deviation that triggered the initial alert is characteristic of "minor image perturbations," in which the image inside the Einstein ring is perturbed by a planet, and therefore a planet is inside the Einstein ring. In this case, 

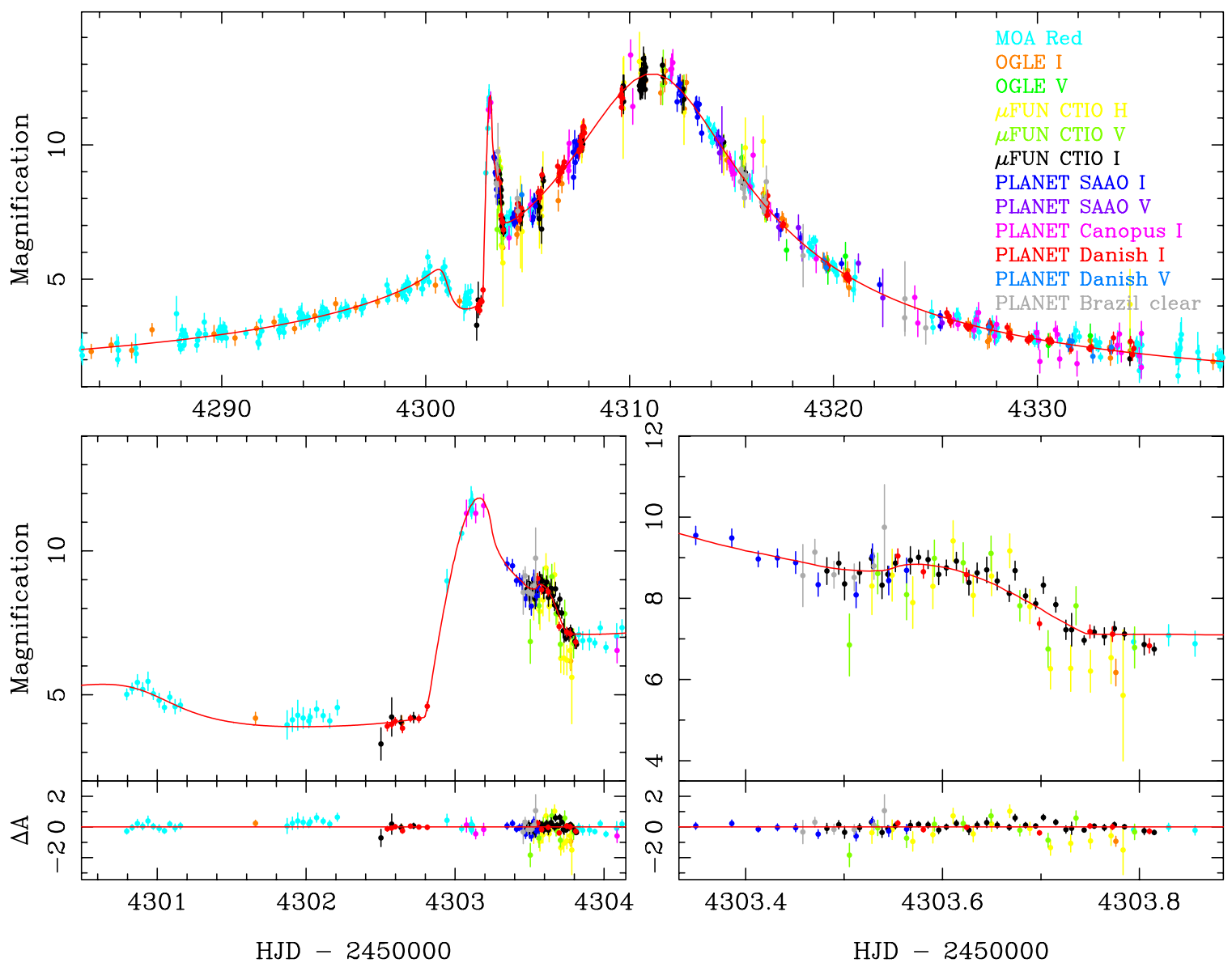

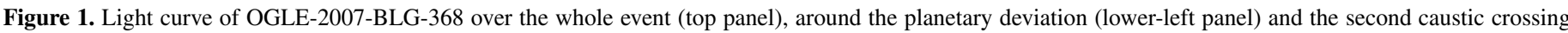

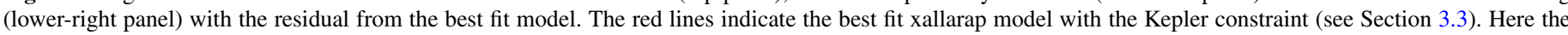

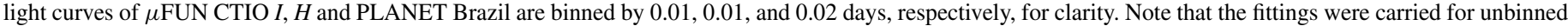
light curves.

two triangular caustics appear near the central caustic, on the opposite side of the planet, as shown in Figure 2. The Danish (La Silla) data show a caustic entrance just prior to their last point, and the MOA and Canopus data confirm this entrance and trace its rise. From these data alone it is clear that the source has passed into the "depression" between the two triangular caustics and then passed over one of the two parallel caustic walls that bound this depressed region. See Figure 2. The subsequent data over the next day trace the path through a triangular caustic. A blind search of parameter space, in which $\chi^{2}$ minimizations were done with various initial parameters, confirms that this is the only viable topology.

In addition to the three single-lens model parameters, the time of peak magnification $t_{0}$, Einstein radius crossing time $t_{\mathrm{E}}$, and the minimum impact parameter $u_{0}$, the standard binary lens model has four more parameters, the planet-host mass ratio $q$, projected separation $d$, the angle of the source trajectory relative to the binary lens axis $\alpha$, and source radius relative to the Einstein radius $\rho=\theta_{*} / \theta_{\mathrm{E}}$, or the source radius crossing time $t_{*}=\rho t_{\mathrm{E}}$. Note that $\rho$ can be used to estimate angular Einstein radius $\theta_{\mathrm{E}}$ by using the source angular radius $\theta_{*}$ which can be estimated from its color and apparent magnitude (Yoo et al. 2004a).

A simple heuristic argument can be given to derive $q, d$, and $\alpha$ from the gross characteristics of the light curve (Gould \& Loeb 1992; Gaudi \& Gould 1997). From the point-lens part of the light curve with the planetary perturbation excluded, we robustly find $t_{0} \simeq 2454311 \mathrm{JD}, t_{\mathrm{E}} \simeq 55$ days, and $u_{0} \simeq 0.08$.

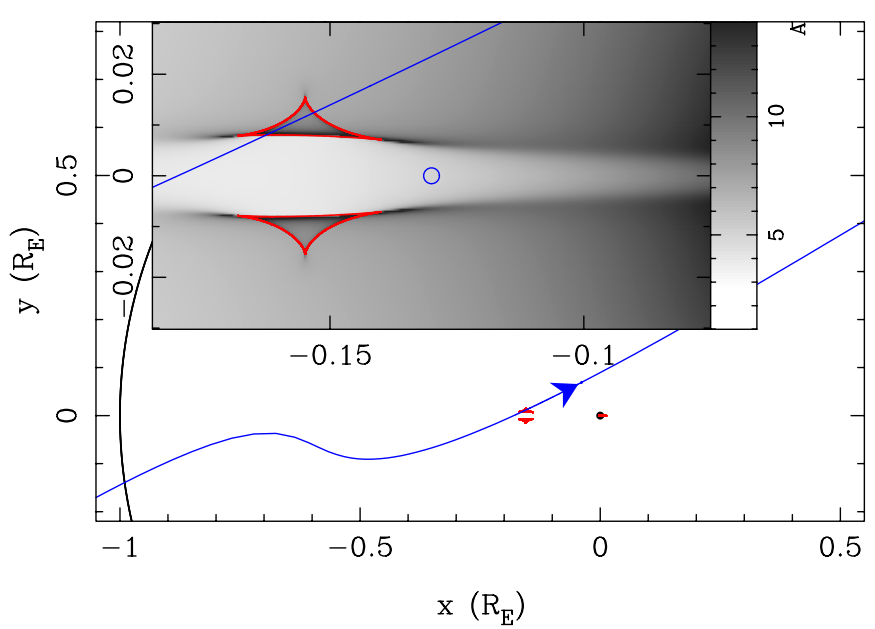

Figure 2. Caustics (red lines) and critical curves (black lines) of OGLE-2007BLG-368 for the best model fitting $\epsilon$ with the Kepler constraint (see Section 3.3). The blue line represents the trajectory of the source. The inset shows the zoom around the planetary caustic crossing, where the gray scale indicates the magnification pattern. The circle in the inset represents the best fit source size.

The time and duration of the planetary deviation is $t_{\mathrm{d}} \simeq 2454303$ $\mathrm{JD}$ and $\Delta t \simeq 1$ day, where we adopt the duration of the negative deviation relative to the single-lens model. By using these planet-model independent values, the position of the planet can be estimated as $d_{-}=\left(\sqrt{u_{\mathrm{d}}^{2}+4}-u_{\mathrm{d}}\right) / 2=0.92$, 
Table 1

Model Parameters

\begin{tabular}{|c|c|c|c|c|c|c|c|c|c|c|c|c|c|c|}
\hline Model & $\begin{array}{c}t_{0} \\
\left(\mathrm{HJD}^{\prime}\right)\end{array}$ & $\begin{array}{c}t_{\mathrm{E}} \\
\text { (days) }\end{array}$ & $u_{0}$ & $\begin{array}{c}q \\
\left(10^{-4}\right)\end{array}$ & $d$ & $\begin{array}{c}\alpha \\
(\mathrm{rad})\end{array}$ & $\begin{array}{c}\rho \\
\left(10^{-3}\right)\end{array}$ & $\pi_{\mathrm{E}}$ & $\begin{array}{c}\phi_{\mathrm{E}} \\
(\mathrm{rad})\end{array}$ & $\xi_{\mathrm{E}}$ & $\begin{array}{c}\phi_{\xi} \\
(\mathrm{rad})\end{array}$ & $\begin{array}{c}P_{\xi} \\
\text { (days) }\end{array}$ & $\epsilon$ & $\chi^{2}$ \\
\hline Standard & 4310.92 & 53.2 & 0.0825 & 1.27 & 0.9227 & 0.452 & 1.88 & $\ldots$ & $\ldots$ & $\ldots$ & $\ldots$ & $\ldots$ & $\ldots$ & 3306.3 \\
\hline$\sigma$ & 0.01 & 0.4 & 0.0008 & 0.02 & 0.0006 & 0.002 & 0.03 & $\ldots$ & $\ldots$ & $\ldots$ & $\ldots$ & $\ldots$ & $\ldots$ & $\ldots$ \\
\hline Parallax & 4311.07 & 59.9 & 0.0765 & 0.77 & 0.9286 & 0.534 & 1.39 & 1.78 & 5.66 & $\ldots$ & $\ldots$ & $\ldots$ & $\ldots$ & 3008.4 \\
\hline$\sigma$ & 0.02 & 1.0 & 0.0011 & 0.04 & 0.0009 & 0.008 & 0.04 & 0.14 & 0.04 & $\ldots$ & $\ldots$ & $\ldots$ & $\ldots$ & $\ldots$ \\
\hline Xallarap* & 4311.10 & 54.1 & 0.0790 & 0.89 & 0.9257 & 0.462 & 1.52 & $\ldots$ & $\ldots$ & 1.73 & 6.08 & 215.9 & $\ldots$ & 2934.9 \\
\hline$\sigma$ & 0.01 & 0.5 & 0.0008 & 0.02 & 0.0007 & 0.002 & 0.03 & $\ldots$ & $\ldots$ & $\ldots$ & $\ldots$ & $\ldots$ & $\ldots$ & $\ldots$ \\
\hline Xallarap*K & 4311.12 & 53.2 & 0.0796 & 0.99 & 0.9252 & 0.438 & 1.61 & $\ldots$ & $\ldots$ & 0.21 & 6.18 & 102.4 & $\ldots$ & 2975.7 \\
\hline$\sigma$ & 0.01 & 0.6 & 0.0010 & 0.02 & 0.0008 & 0.002 & 0.03 & $\ldots$ & $\ldots$ & $\ldots$ & $\ldots$ & $\ldots$ & $\ldots$ & $\ldots$ \\
\hline Xallarap & 4311.08 & 57.7 & 0.0781 & 0.85 & 0.9266 & 0.516 & 1.46 & $\ldots$ & $\ldots$ & 0.35 & 6.20 & 103.0 & 0.48 & 2919.0 \\
\hline$\sigma$ & 0.01 & 0.6 & 0.0009 & 0.02 & 0.0007 & 0.002 & 0.03 & $\ldots$ & $\ldots$ & $\ldots$ & $\ldots$ & $\ldots$ & $\ldots$ & $\ldots$ \\
\hline Xallarap $^{K}$ & 4311.12 & 55.4 & 0.0793 & 0.95 & 0.9255 & 0.478 & 1.55 & $\ldots$ & $\ldots$ & 0.16 & 4.73 & 106.3 & 0.77 & 2936.9 \\
\hline$\sigma$ & 0.01 & 0.5 & 0.0007 & 0.02 & 0.0006 & 0.002 & 0.03 & $\ldots$ & $\ldots$ & $\ldots$ & $\ldots$ & $\ldots$ & $\ldots$ & $\ldots$ \\
\hline$\sigma_{\text {systematic }}$ & 0.01 & 2.3 & 0.0022 & 0.21 & 0.0019 & 0.039 & 0.21 & $\ldots$ & $\ldots$ & $\ldots$ & $\ldots$ & $\ldots$ & $\ldots$ & $\ldots$ \\
\hline
\end{tabular}

Notes. Standard model includes neither parallax nor xallarap effects. HJD' $=$ HJD-2450000. Models with superscripts “*” and " $K$ ” indicate fixed $\epsilon=0$ and Kepler constraint $M_{s}=M_{c}=1 M_{\odot}$, respectively. The lines with " $\sigma$ " list the $1 \sigma$ error of parameters given by MCMC, for which the xallarap parameters are fixed at the best values for the xallarap models because xallarap parameters are strongly degenerate and it is hard to satisfy the convergence criteria. $\sigma_{\text {systematic }}$ indicates the systematic errors (see Section 4).

where $u_{\mathrm{d}}=\sqrt{\tau\left(t_{\mathrm{d}}\right)^{2}+u_{0}^{2}}=0.166$ and $\tau\left(t_{\mathrm{d}}\right)=\left(t_{\mathrm{d}}-t_{0}\right) / t_{\mathrm{E}}$. The angle of the source trajectory relative to the binary lens axis, $\alpha$, can be given by $\sin \alpha=u_{0} / u_{\mathrm{d}}=0.48$, therefore $\alpha=0.5 \mathrm{rad}$. The separation of the two triangular caustics is given by $d_{\text {caus }}=2(\gamma-1)^{1 / 2}$ in the unit of the planet angular Einstein radius $\theta_{p}=q^{1 / 2} \theta_{\mathrm{E}}$ (Schneider et al. 1992), where $\gamma=d_{-}^{-2}$ is the shear. The duration required to pass the "depression" between the two triangular caustics is given by $\Delta t=2(\gamma-1)^{1 / 2} q^{1 / 2}(\csc \alpha) t_{\mathrm{E}}$. Therefore, we find that the planet has the sub-Saturn mass ratio, $q \sim 1 \times 10^{-4}$. From the light curve around $\mathrm{JD}=2454303$, we can roughly find the time it takes the source radius to cross the caustic $t_{\text {cross }} \simeq 0.23$ days. Therefore, the source radius crossing time can be estimated as $t_{*}=t_{\text {cross }} \sin \alpha \sim 0.1$ day. These first-order estimates of the planetary modeling are very robust. The actual lightcurve modeling will investigate several higher-order effects and possible systematics, but all within the context of the topology defined by Figure 2, and the main conclusions remain robust.

The light-curve modeling was done by two independent codes. One uses the hybrid point-source, individual image rayshooting method of Bennett (2009), which has been developed from the first completely general finite-source binary lens calculations of Bennett \& Rhie (1996). The other uses the same basic strategy, but was independently written by MOA. The best fit binary lens model was found by the Markov Chain Monte Carlo (MCMC) method (Verde et al. 2003). The Markov chains of preliminary runs were used to derive the optimal directions and step sizes for exploring parameter space. The resultant distribution of the chains gives us the best fit parameters and their errors. We use a linear limb-darkening model for the source star using the coefficients, $u=0.5250$ for $I$-band, 0.6834 for $V, 0.3434$ for $H$ and $u=0.566$ for MOA-Red which is a mean of $R$ and $I$, from Claret (2000) for a G6 type source star with $T=5750 \mathrm{~K}$ and $\log g=4$, which is based on the best fit source $V-I$ color (see Section 5). The best fit source and blend are plotted in the color-magnitude diagram (CMD; Figure 3). The best fit standard binary lens model has a planetary mass ratio of $q=1.3 \times 10^{-4}$ and other parameters as listed in Table 1, in which $q, d$ and $\alpha$ the source radius crossing time, $t_{*}=$ $\rho t_{\mathrm{E}}=0.1$ day are consistent with the first order estimates given above.

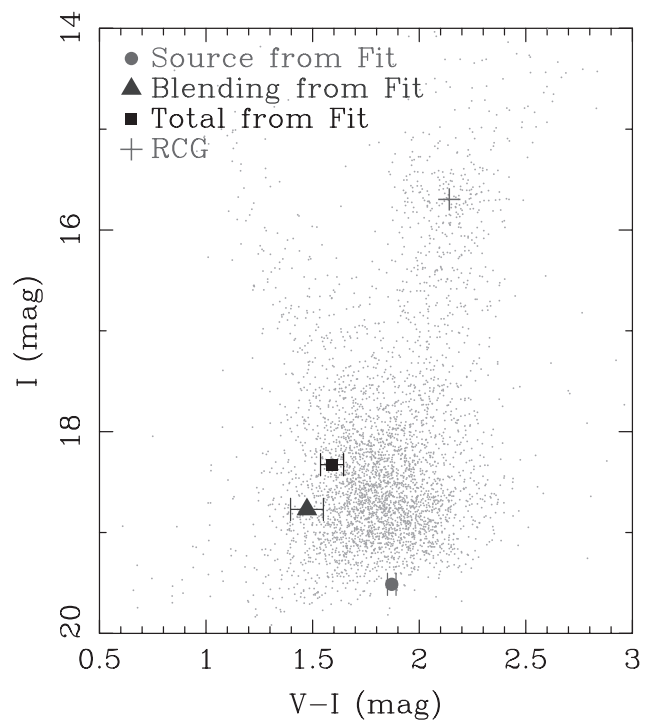

Figure 3. OGLE $(V-I, I)$ color-magnitude diagram around OGLE-2007-BLG368. The filled circle and triangle indicate the source and blended light from the fit, respectively. The filled square represents the total flux of the source and blend. Here the errors in $I$ are too small to be visible. The cross indicates the center of the RCG.

However, the overall light curve shows asymmetric residuals about the primary peak, which suggests either the microlensing parallax effect (Gould 1992; Alcock et al. 1995; Smith et al. 2002) by which the Earth's orbital motion distorts the light curve and/or the xallarap effect, which is a similar distortion caused by the orbital motion of a binary source (Griest \& Hu 1992; Han \& Gould 1997). Therefore, we compare the data to models that included parallax and xallarap.

\subsection{Microlensing Parallax}

The parallax effect is represented by two additional parameters, an amplitude $\pi_{\mathrm{E}}=\pi_{\text {rel }} / \theta_{\mathrm{E}}$, i.e., the lens-source relative parallax $\pi_{\text {rel }}=\left(\pi_{1}-\pi_{\mathrm{s}}\right)$ in unit of the angular Einstein radius $\theta_{\mathrm{E}}=R_{\mathrm{E}} / D_{l}$, and a direction of the relative source-lens proper motion relative to north toward east $\phi_{\mathrm{E}}$, where $D_{l}$ is the distance to the lens. As shown in Table 1, the best fit parallax model 
improves $\chi^{2}$ by $\Delta \chi^{2}=298$ relative to the best standard binary model. If this parallax model were the correct model, we could derive the lens mass $M=\theta_{\mathrm{E}} /\left(\kappa \pi_{\mathrm{E}}\right)=0.040 \pm 0.005 M_{\odot}$, and distance $D_{l}=\mathrm{AU} / \pi_{l}=867 \pm 93 \mathrm{pc}$, for this model of the lens (Gould 1992). Here $\kappa=4 G / c^{2} \mathrm{AU}=8.144$ mas $M_{\odot}^{-1}$ (milliarcsec per solar mass) and we have assumed the source distance $D_{s}=\mathrm{AU} / \pi_{\mathrm{s}}=8.0 \pm 1.4 \mathrm{kpc}$ where the error is based on $17 \%$ standard deviation in the Galactic bar model (Han \& Gould 2003). This model implies that the lens is a nearby brown dwarf. However, as shown in the next section, the best xallarap model yields a significantly better $\chi^{2}$, with an improvement by $\Delta \chi^{2}=89.4$. Furthermore, if the signal were due to parallax, we should have found the best xallarap model with the same (R.A. $\xi$, decl. $\xi$ ) values as the celestial coordinates of the source as seen from the Earth (R.A., decl. $)=\left(269^{\circ} .1,-32^{\circ} .2\right)$ when its period of the source orbital motion, eccentricity, and perihelion, celestial pole are fixed as the values of Earth's orbit. However, we obtained the best model with (R.A. $\xi$, decl. $\xi)=\left(309^{\circ} .4\right.$, $-24.0) \pm(0.5,0.2)$, which is inconsistent with the expected values for parallax. We conclude that this distortion is not likely due solely to parallax.

\subsection{Xallarap}

If the orbit is assumed to be circular, and the companion assumed to generate negligible flux compared to the source, the xallarap effect can be represented by five additional parameters, an amplitude, $\xi_{\mathrm{E}}=a_{s} / \hat{r}_{\mathrm{E}}$, that is the semimajor axis of the source's orbit, $a_{s}$, in the unit of the Einstein radius projected on the source plane, $\hat{r}_{\mathrm{E}}=R_{\mathrm{E}} D_{s} / D_{l}$, the direction of the relative source-lens proper motion, $\phi_{\xi}$, the direction of observer relative to the source orbital axis, R.A. $\xi$ and decl. $\xi$, orbital period, $P_{\xi}$. For an elliptical orbit, two additional parameters are required, the orbital eccentricity, $\epsilon$ and time of perihelion, $t_{\text {peri }}$.

The best xallarap model, with $\epsilon$ fixed at $\epsilon=0$ and with $\epsilon$ as a free parameter, improved $\chi^{2}$ by $\Delta \chi^{2}=74$ and 89 , respectively, relative to the best parallax model. The best fit parameters are listed in Table 1. We also fitted models with a bright binary companion, but in every case, the dark binary companion provided the best fit. Therefore, we keep only models having companions with negligible flux compared to the source in the following analysis, which would be appropriate for a white dwarf or M dwarf companion.

In Figure 4, we show the $\chi^{2}$ of the best xallarap models as a function of $P_{\xi}$ with orbital eccentricity fixed at $\epsilon=0$ and fitting for $\epsilon$. One can see that xallarap models are significantly better than the best parallax model, and that the xallarap model in which $\epsilon$ is a free parameter is slightly better than the model fixing $\epsilon=0$. For the xallarap models, $\chi^{2}$ is flat for $P_{\xi} \geqslant 150$ days, in which regime $P_{\xi}$ and $\xi_{\mathrm{E}}$ are strongly degenerate.

Of course, every microlensing event must have a non-zero microlensing parallax, but the addition of parallax to these xallarap models did not provide a significant $\chi^{2}$ improvement. The parallax and xallarap parameters are highly degenerate and tend to complicate the analysis, so we have excluded parallax from most of our xallarap models.

\subsection{Xallarap with the Kepler Constraint}

In Section 3.2, the model with the lowest $\chi^{2}$ is the xallarap model with non-zero $\epsilon$. However, this model leads to a xallarap amplitude of $\xi_{\mathrm{E}}=0.35$, which is larger than would be induced by a "normal" main-sequence companion, where $\xi_{\mathrm{E}}$

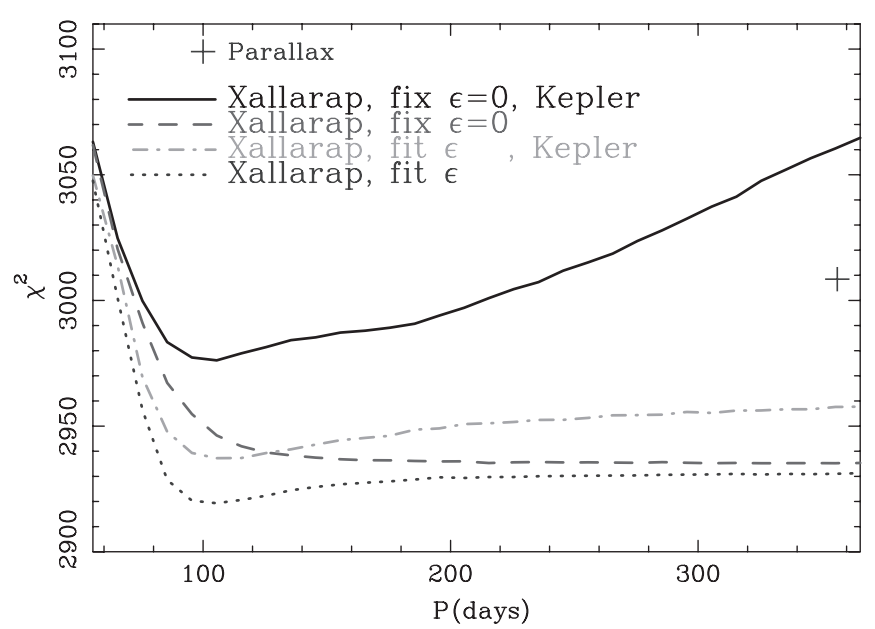

Figure 4. $\chi^{2}$ of the best xallarap model as a function of the orbital period of the source star and its companion. The solid and dashed lines indicate the model with fixed orbital eccentricity $\epsilon=0$ with and without the Kepler constraint, respectively. The dot-dashed and dotted lines indicate the model allowing a free-fit of $\epsilon$ subject and not subject to the Kepler constraint, respectively. The best parallax model is plotted as a " + " for comparison.

is expressed, making use of Kepler's third law, by

$$
\xi_{\mathrm{E}}=\frac{a_{s}}{\hat{r}_{\mathrm{E}}}=\frac{1 \mathrm{AU}}{\hat{r}_{\mathrm{E}}}\left(\frac{M_{c}}{M_{\odot}}\right)\left(\frac{M_{\odot}}{M_{c}+M_{s}} \frac{P_{\xi}}{1 \mathrm{yr}}\right)^{\frac{2}{3}} .
$$

From this equation and parameters for this model, the lower limit of the companion mass to the source is given by

$$
M_{c} \geqslant \xi_{\mathrm{E}}^{3} \hat{r}_{\mathrm{E}}^{3}\left(\frac{P_{\xi}}{1 \mathrm{yr}}\right)^{-2} \sim 50 M_{\odot},
$$

which would imply a black hole companion, since a $50 M_{\odot}$ star would exceed our upper limit on the brightness of a companion to the source by more than 5 mag.

Black holes are quite rare compared to stars, so we should consider the prospect of a more normal stellar companion. We can use Kepler's third law, the projected Einstein radius, $\hat{r}_{\mathrm{E}}$, source mass, $M_{s}$, and the source companion mass, $M_{c}$, to constrain the magnitude of the xallarap vector $\xi_{\mathrm{E}}$ (Bennett et al. 2008; Dong et al. 2009b). From Section 5, we derive $M_{s}=0.9 \pm 0.1 M_{\odot}$ and assume a white dwarf companion $M_{c}=1.0 \pm 0.4 M_{\odot}$ (which would be the most massive dark companion with a plausible a priori probability). Inserting these masses and other relevant parameters into Equation (1), the maximum allowed $\xi_{\mathrm{E}}$ for the best xallarap models for the circular orbit xallarap and non-zero $\epsilon$ models are given by

$$
\xi_{\mathrm{E}, \max }=0.11 \pm 0.04(\epsilon=0) \text { and } 0.06 \pm 0.02(\epsilon \text { free }),
$$

where the error is estimated from the errors in $\theta_{*}, M_{s}$, and $M_{c}$.

Because our best fit values of $\xi_{\mathrm{E}}=1.73$ and 0.35 for the circular orbit and non-zero $\epsilon$ are much larger than $\xi_{\mathrm{E} \text {, max }}$ given above, they are inconsistent with our upper limit on the source companion mass. To find the best xallarap model that is allowed by Kepler's third law, we have done MCMC runs with an additional constraint contribution to $\chi^{2}$ given by

$$
\chi_{\mathrm{orb}}^{2}=\Theta\left(\xi_{\mathrm{E}}-\xi_{\mathrm{E}, \max }\right)\left(\frac{\xi_{\mathrm{E}, \max }-\xi_{\mathrm{E}}}{\sigma_{\xi_{\mathrm{E}, \max }}}\right)^{2} .
$$

where $\xi_{\mathrm{E}, \max }$ is evaluated by Equation (1) with parameters in each step of the MCMC and fixed values of $M_{s}=M_{c}=1 M_{\odot}$ 
and $50 \%$ error in $\xi_{\mathrm{E}, \max }$, which depend only weakly on other parameters. Here, $\Theta$ is the Heaviside step function.

In Table 1, we show the best fit model parameters and $\chi^{2}$ for the circular orbit and non-zero $\epsilon$ cases. In Figure 4, we show the $\chi^{2}$ of the best-fit xallarap models with the Kepler constraint as a function of $P_{\xi}$. One can see that if we impose the Kepler constraint, the xallarap solution with $\epsilon$ free is better than the case of fixed $\epsilon=0$ by $\Delta \chi^{2}=39$. Although this $\chi^{2}$ is worse, by $\Delta \chi^{2}=18$, than the model without the Kepler constraint, this is the best model that is allowed for a plausible companion mass.

\section{THE ERRORS IN PARAMETERS WITH SYSTEMATICS}

We have investigated second-order effects to explain the clear asymmetry about the peak in Sections 3.1, 3.2, and 3.3. We also searched for models with an additional mass besides the lens star and planet, but neither an additional stellar or planetary companion to the lens star could account for observed lightcurve asymmetry.

We are sure that there is an asymmetry in the light curve, because we see qualitatively similar trends in both MOA and OGLE light curves as shown in Figure 5, which have different typical seeing and were reduced by independent pipelines. However, we are not fully confident that this xallarap amplitude is correct because of the unphysically large $\xi_{\mathrm{E}}$ and an additional factor: there is a bright red clump giant (RCG) star with $(V-I, I)=(2.01,15.56)$ at the northeast corner of the Keck AO image (see details in Section 6) in Figure 6, which is only 1".1 away from the source. The wing of the giant star PSF interferes with photometering the source on the OGLE images, with typical seeing of $\sim 1^{\prime \prime}$. 2 , and even worse in the MOA images, with typical seeing of $\sim 2$ ". 0 . The differential atmospheric extinction and refraction may cause systematic asymmetry on the light curve. Here the differential refraction causes the positional shift of the target on the sky, which generates residuals on the subtracted images in DIA. These effects depend on the color of stars and air mass. The mean air mass changes slowly during the event because the mean elevation of the target changes in season. They can generally be reduced by choosing the reference stars with the similar color as the target for aligning the image coordinates and solving the kernel in DIA. However, the effects due to the blending star with different color from the target are hard to remove. In particular, subtle effects on the bright blending star can cause significant effects on the faint target. So the photometry of this event is challenging. We tested the modeling with data points taken at air mass $>1.3$ removed, but this does not make any significant difference. When we model by first removing either the OGLE or MOA data set, the results are qualitatively unchanged.

As argued in Section 3, the planetary deviation is clearly detected, and the planet parameters can be estimated robustly by simple inspection. Although our analysis of the parallax and xallarap fits indicate the presence of unrecognized systematic errors in the data, these errors do not affect these basic inferences about the planet itself. Therefore, we are only interested in robustly estimating the parallax or xallarap parameters to the extent that they can provide additional information about the lens. However, having discarded the parallax model for the asymmetry, the xallarap parameters themselves provide no new information about the lens, and are therefore of no intrinsic interest to us. We therefore do not further investigate the cause of the systematics in the light curve, and instead seek only to determine the (relatively minor) extent to which these systematics affect our precise determination of the planetary parameters. To
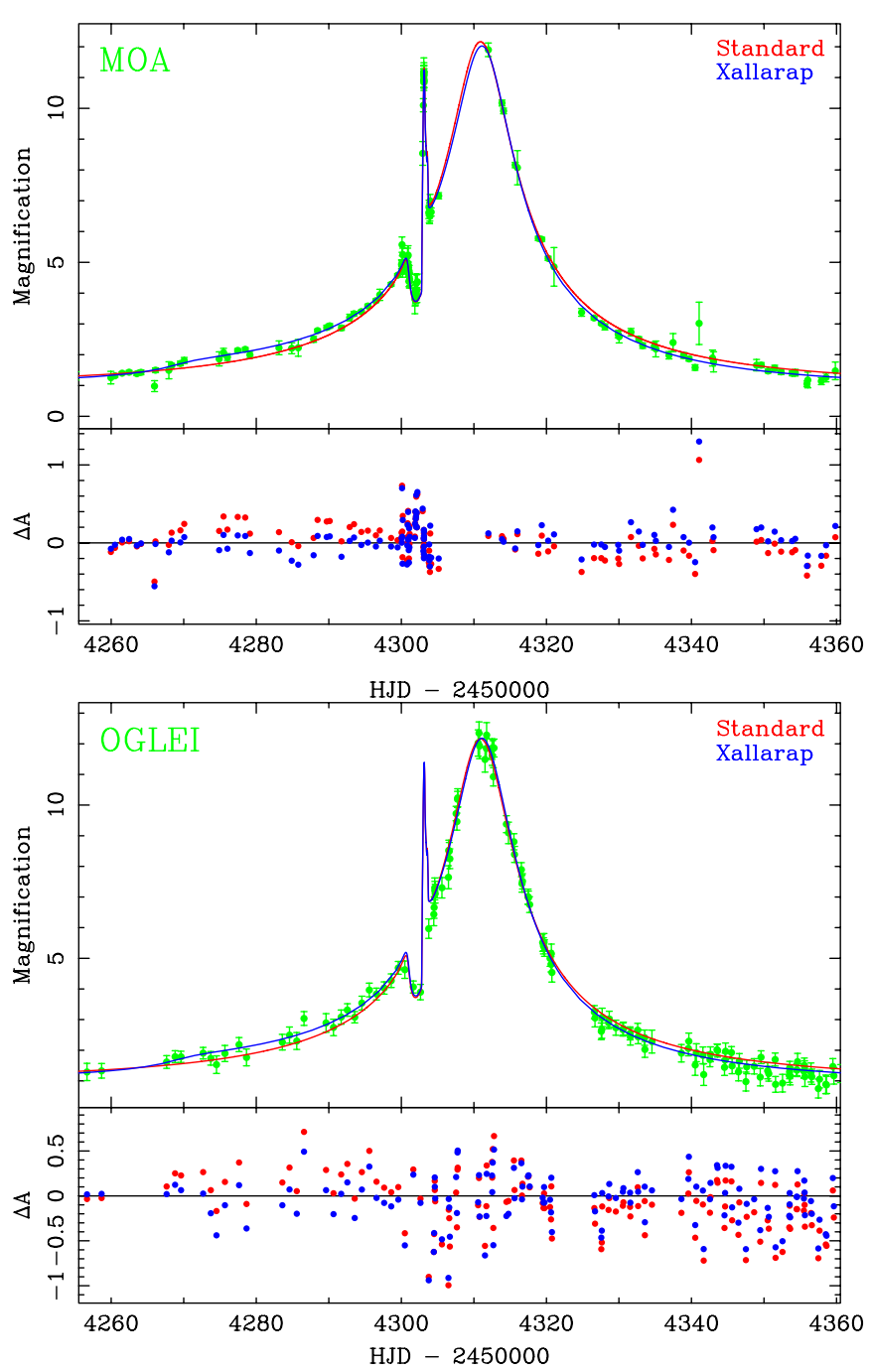

Figure 5. Light curves of MOA-Red (top panel) and OGLE I (bottom panel) with the best standard (red line and residual) and xallarap models with $\epsilon$ being fit subject to the Kepler constraint (blue line and residual). Here MOA data are binned by 1 day outside of the planetary signal at JD-2450000 $=4300-4304$. We can see a similar asymmetry in both light curves which are well fitted by the xallarap model in both cases.

do this, we consider the standard and all the possible xallarap models shown in Table 1 as viable, and take the differences of the parameters as the size of the systematic errors. We take parameters of the xallarap model with non-zero $\epsilon$ and the Kepler constraint (indicated as Xallarap ${ }^{K}$ in Table 1), as the median. The resultant systematic errors are listed in Table 1 and $I_{\mathrm{s}, \mathrm{OGLE}}=19.51 \pm 0.03 \mathrm{mag}$ and $I_{\mathrm{b}, \mathrm{OGLE}}=18.77 \pm 0.02 \mathrm{mag}$. The values and errors in the following sections are estimated taking these systematic errors into account.

\section{SOURCE STAR CHARACTERIZATION}

We must determine the source star angular radius, $\theta_{*}$, in order to determine the angular Einstein radius, $\theta_{E}$, from the light-curve parameters. Since we do not have infrared light-curve data of high enough quality to accurately measure the source brightness in the infrared (Gould et al. 2009; Bennett et al. 2009), we use the two-filter method of Yoo et al. (2004a) to determine $\theta_{*}$. The values and errors stated in this section are the final values including systematic errors, as they are subsequently reestimated after Section 4. However, these are qualitatively the 


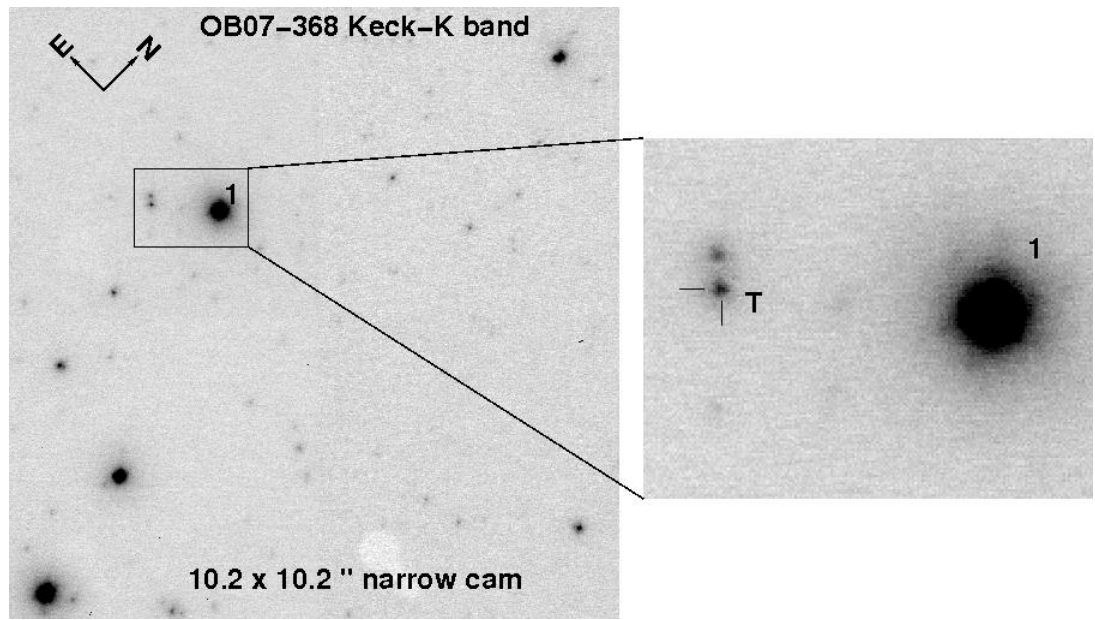

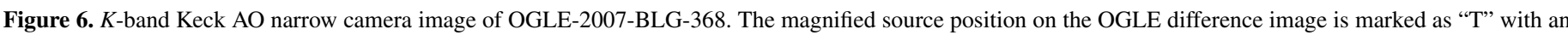

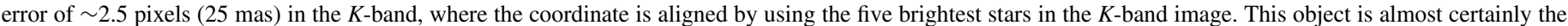

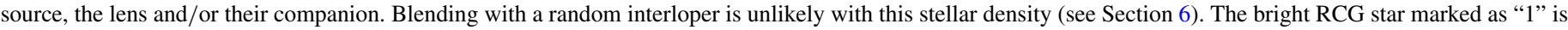
1 1". 1 way from the source, whose PSF tail covers the source on OGLE image with typical seeing of 1".2.

same within the errors as the original results used for the initial light-curve modeling in Section 3.

\subsection{Extinction Correction}

The $V$ and $I$ magnitudes of the source star from the lightcurve fit (see Section 3) must be corrected for the extinction and reddening due to the interstellar dust to infer the spectral type of the source. Because this field is out of the OGLE-II extinction map (Sumi 2004), we estimate the extinction and reddening to the source by using RCG, which are known to be an approximate standard candle (Stanek et al. 1997; Paczyński \& Stanek 1998). Figure 3 shows the calibrated OGLE CMD in $3 \times 3 \operatorname{arcmin}^{2}$ field around the event $[(l, b)=(358.3488,-3.6861)]$. From this CMD, we find the RCG centroid:

$$
(V-I, I)_{\mathrm{RC}, \mathrm{obs}}=(2.14,15.70),
$$

where the errors are negligible comparing to the intrinsic error in the RCG centroid, as described below.

We adopt the intrinsic RCG magnitude $M_{I, \mathrm{RC}, 0}=-0.25 \pm$ $0.05, M_{V, \mathrm{RC}, 0}=0.79 \pm 0.08$ and color $(V-I)_{\mathrm{RC}, 0}=1.04 \pm$ 0.08 from Bennett et al. (2008) which is based on Girardi \& Salaris (2001) and Salaris \& Girardi (2002), where the error is assigned based on the size of the theoretical corrections to the RCG magnitudes. Taking account of the bar structure of the Galactic bulge, the offset of the distance modulus (DM) between the GC that is assumed to be at $8.0 \pm 0.5 \mathrm{kpc}$ (Reid 1993) and the average stars in the field have $\triangle \mathrm{DM}=0.00 \pm 0.05$ (Nishiyama et al. 2005). So the dereddened RCG centroid in the field is expected to be

$$
(V-I, I)_{\mathrm{RC}, 0}=(1.04,14.27) \pm(0.08,0.15) .
$$

Comparing these centroids (Equations (5) and (6)), we find the average reddening and extinction in this field is

$$
\left(E(V-I), A_{I}\right)=(1.10,1.43) \pm(0.08,0.15),
$$

where $R_{V I}=A_{V} / E(V-I)=2.30$, which corresponds to $R_{V}=2.64$ (Cardelli et al. 1989). Applying this average extinction to this event, the source's $(V-I, I)_{\mathrm{s}, \text { OGLE }}$ from fitting of the well calibrated OGLE $V$ and $I$ light curve and the dereddened source magnitude and color $(V-I, I)_{\mathrm{s}, 0}$ are

$$
\begin{gathered}
(V-I, I)_{\mathrm{s}, \text { OGLE }}=(1.85,19.51) \pm(0.06,0.03), \\
(V-I, I)_{\mathrm{s}, \text { OGLE }, 0}=(0.75,18.08) \pm(0.10,0.16) .
\end{gathered}
$$

Independently, the dereddened source color, $(V-I)_{\mathrm{s}, \mathrm{CTIO}, 0}=$ $0.77 \pm 0.02 \pm 0.08$, is estimated by comparing $(V-I)_{\mathrm{RC}, 0}$, the CTIO RCG color and the CTIO source color $(V-I)_{\mathrm{s}, \mathrm{CTIO}}$ which is given by the model-independent regression of CTIO $V$ and $I$ light curves. This is consistent with $(V-I)_{\mathrm{s}, \mathrm{OGLE}, 0}$, but more accurate. In the following analysis, we adopt the value

$$
(V-I, I)_{\mathrm{s}, 0}=(0.77,18.08) \pm(0.08,0.16),
$$

which implies that the source is a mid-G star in the bulge (Bessell $\&$ Brett 1988) with mass of $M_{s}=0.9 \pm 0.1 M_{\odot}$ (Schmidt-Kaler 1982). The reddened $(V-I, I)_{\mathrm{s}}$ is plotted in Figure 3 .

The dereddened blended light from the light curve is

$$
(V, I)_{\mathrm{b}, 0}=(17.71,17.34) \pm(0.18,0.15) .
$$

Note that if this blended light is from the lens or companion of the lens, these values may be over-corrected for extinction because these objects are in front of the source. Thus, these magnitudes can be used as an upper limit on the combined light of the lens, any companion of the lens, and the source in the following analysis.

\subsection{Source Star Angular Radius}

Following Yoo et al. (2004a), the dereddened source color and brightness $(V-K, K)_{\mathrm{s}, 0}=(1.69,17.16)$ are estimated using the observed $(V-I, I)_{\mathrm{s}, 0}$ as given by Equation (10) and the color-color relation (Bessell \& Brett 1988). By using this $(V-K, K)_{\mathrm{s}, 0}$ and the empirical color/brightness-radius relation by Kervella et al. (2004), we estimate the source angular radius, $\theta_{*}=0.81 \pm 0.07 \mu$ as, where the error includes uncertainty in the color conversion and the color/brightnessradius relation. On the other hand, $(V-I, I)_{\mathrm{s}, 0}$ and optical color/brightness-radius relation by Kervella \& Fouqué (2008) 

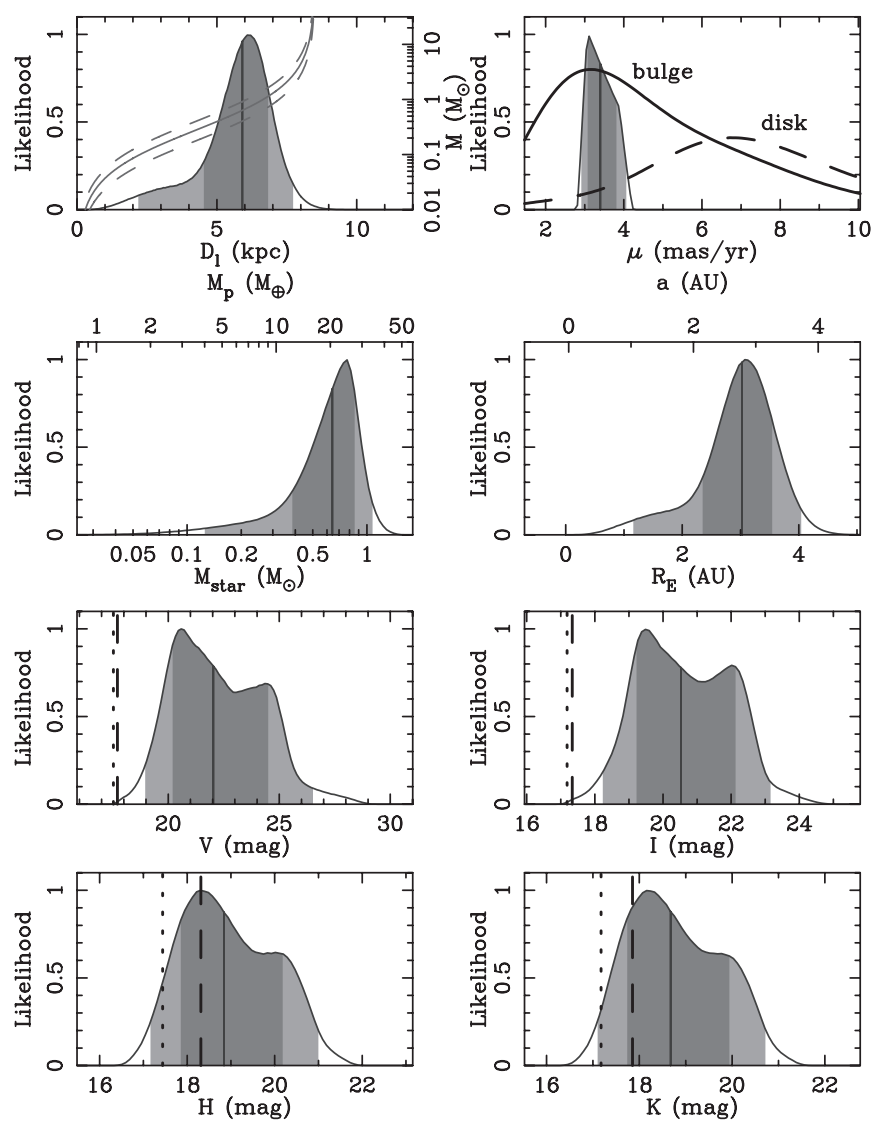

Figure 7. Probability distributions from a Bayesian analysis for the distance, $D_{l}$, transverse velocity, $v_{\mathrm{t}}$, mass, $M_{\mathrm{star}}$, Einstein radius $\left(R_{\mathrm{E}}\right), V, I, H$, and $K$-band magnitudes of the primary star of the lens system. The vertical solid lines indicate the median values. The dark and light gray shaded regions indicate the $1 \sigma$ and $2 \sigma$ limits. The gray solid and dashed curves in the top-left panel indicate the mass-distance relation of the lens from the measurement of $\theta_{\mathrm{E}}$ with $1 \sigma$ errors, respectively, assuming $D_{s}=8 \mathrm{kpc}$. Note $D_{s}$ is not fixed in the actual Bayesian analysis. Thick solid and dashed lines in the top-right panel represent the typical $\mu$ distributions of the bulge and disk lens populations, respectively. The vertical dashed and dotted lines in the $V, I, H$, and $K$-band panels represent observed upper limit and $1 \sigma$ error, respectively.

yields $\theta_{*}=0.83 \pm 0.05 \mu$ as, which is consistent with above. We adopt the mean of these estimates,

$$
\theta_{*}=0.82 \pm 0.07 \mu \text { as. }
$$

The angular and projected Einstein radii, and lens-source relative proper motion $\mu$ are estimated, respectively, as

$$
\begin{gathered}
\theta_{\mathrm{E}}=\frac{\theta_{*}}{\rho}=529 \pm 84 \mu \mathrm{as}, \\
\hat{r}_{\mathrm{E}}=\theta_{E} \times D_{s}=[4.2 \pm 0.7]\left(\frac{D_{s}}{8 \mathrm{kpc}}\right) \mathrm{AU} . \\
\mu=\frac{\theta_{E}}{t_{\mathrm{E}}}=3.5 \pm 0.6 \mathrm{mas} \mathrm{yr}^{-1} .
\end{gathered}
$$

As shown in the top right panel of Figure 7, the measured value

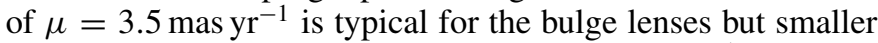
than the typical value for disk lenses, 5-10 mas yr $^{-1}$, although it is not inconsistent with a disk lens.

\section{KECK AO OBSERVATION}

$H$ and $K$ AO images of the event were taken by the Keck telescope at HJD $=2454332.77689$ and 2454332.77977, respectively. The magnification at the time of the Keck images are taken is $A_{\text {Keck }}=2.490$. The magnified source position on the OGLE difference image is marked with the error of $\sim 2.5$ pixels ( 25 mas) in the $K$-band. From the Keck $K$-band image, the density of ambient stars with $3 \sigma$ detection limit that correspond to $K \leqslant 18.1 \mathrm{mag}$, is $\sim 0.3 \mathrm{arcsec}^{-2}$. We conservatively assume that the separation of two stars must be more than the measured FWHM of the PSF of 0.08 arcsec in order to be separately resolved. Therefore, the probability of blending with any random interloper, that is not related with this event, is only $\sim 0.6 \%$, implying this object is almost certainly the source, the lens and/ or their companion. The $H$ and $K$ magnitude were measured by PSF photometry and calibrated to the Two Micron All Sky Survey (2MASS) system using the $H$ and $K$ images taken by the IRSF telescope in South Africa, following the method in Janczak et al. (2010),

$$
(H, K)_{\mathrm{s}, \mathrm{Keck}}=(16.53,16.23) \pm(0.03,0.02),
$$

and the magnitudes corrected for extinction given by $A_{H} / A_{V}=$ 0.176 and $A_{K} / A_{V}=0.105$, which are estimated by using Cardelli et al. (1989)'s law with the $R_{V}=2.64$ measured above, are

$$
(H, K)_{\mathrm{s}, 0, \text { Keck }}=(16.09,15.96) \pm(0.04,0.03) .
$$

The $I-H$ and $I-K$ source colors are estimated from ( $V-$ $I)_{s, 0}$ given by light-curve fitting (Equation (10)) by using the color-color relations of Bessell \& Brett (1988),

$$
(I-H, I-K)_{\mathrm{s}, 0}=\left(0.86_{-0.12}^{+0.11}, 0.92_{-0.13}^{+0.12}\right) .
$$

Therefore, $H$ and $K$ source magnitudes are given as

$$
(H, K)_{\mathrm{s}, 0}=\left(17.23_{-0.19}^{+0.20}, 17.16 \pm 0.20\right) .
$$

Then, the magnitude of the source when the Keck images were taken are

$$
\begin{aligned}
& (H, K)_{\mathrm{s}, 0}-2.5\left(\log \left[A_{\text {Keck }}\right], \log \left[A_{\text {Keck }}\right]\right) \\
& =\left(16.23_{-0.19}^{+0.20}, 16.17 \pm 0.20\right) .
\end{aligned}
$$

By subtracting Equation (20) from Equation (17), we have the magnitude of the lens and/or companion of the lens or source, which serve as an upper mass limit of the lens

$$
(H, K)_{l, \max , 0}=\left(18.3_{-0.9}^{+\infty}, 17.9_{-0.7}^{+\infty}\right) .
$$

This $K$-band magnitude implies that the upper limit of the lens is an early $\mathrm{G}$ dwarf with mass of $M_{l, \max }=1.0_{-\infty}^{+0.2} M_{\odot}$ from (Schmidt-Kaler 1982; Bessell \& Brett 1988). These $H$ and $K$-band upper limits are used for constraining lens star in Section 7. If we could obtain a second epoch $\mathrm{AO}$ observation that gave us the baseline photometry, we would be able to constrain $(H, K)_{l, \text { max }, 0}$, much better.

For other (brighter) events, we have found that the $H$-band source magnitude estimated by fitting the CTIO $H$-band light curve gives a more precise value for the $H$ magnitude of the source. But when we attempt such an analysis for this event, we find significant indications of systematic errors. This is not very surprising because this target does not reach high magnification and is heavily blended with a nearby bright star. Also, because of the bright infrared sky brightness, the CTIO $H$-band images do not go as deep as the optical images. Therefore, we do not use this CTIO $H$-band source magnitude in the following analysis. 


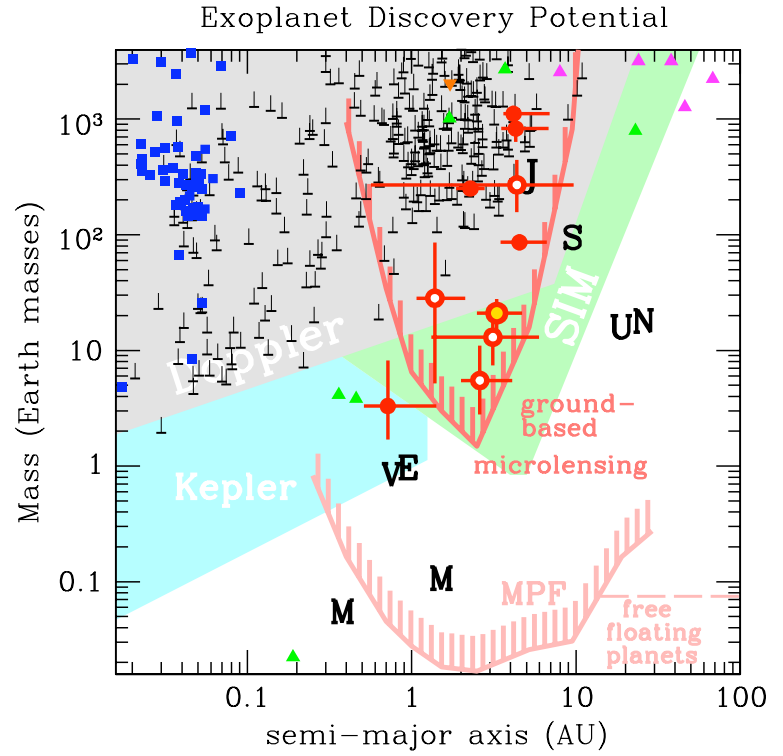

Figure 8. Known exoplanets as a function of mass vs. semimajor axis, along with the predicted sensitivity curves for various methods. The red filled and open circles with error bars indicate the microlensing planets with mass measurements and mass estimated by Bayesian analysis, respectively (see Section 7.2). OGLE2007-BLG-368Lb is indicated by the gold-filled open circle. The blue dots represent the planets first detected via transit. The black bars with upwardpointing error bars (indicating $1 \sigma \sin i$ uncertainty) are the radial velocity planet detections. The green and magenta triangles indicate the planets found by timing (including the pulsar planets) and by direct detection, respectively. The yellow, cyan, and light green shaded regions indicate the expected sensitivity limits of the radial velocity, Kepler and SIM space missions. The red and pink curves indicate the predicted lower sensitivity limits for a ground-based and spacebased (Bennett \& Rhie 2002) microlensing planet search program, respectively. The solar system's planets are indicated with black letters.

\section{LENS SYSTEM MASSES AND DISTANCE}

The lens system mass, $M_{l}$, distance, $D_{l}$, and lens-source relative velocity are directly constrained by only two measured parameters, the Einstein radius crossing time, $t_{\mathrm{E}}$, and the angular Einstein radius, $\theta_{E}$. However, we can further constrain them by a Bayesian analysis using a model of Galactic kinematics (Alcock et al. 1995; Beaulieu et al. 2006; Gould et al. 2006; Bennett et al. 2008). The mass of the planet can be determined to the same precision as $M_{l}$ because the uncertainty in the mass ratio, $q$, is much smaller than the uncertainty in the Bayesian estimate of $M_{l}$.

\subsection{Planetary System Parameter for OGLE-2007-BLG-368Lb}

For this event, we observed finite source effects from which we measured the angular Einstein radius $\theta_{\mathrm{E}}$ (Equation (13)), or equivalently the proper motion $\mu$ (Equation (15)), of the lens system. So we can break one link of the three-fold degeneracy by the relation,

$$
\theta_{E}^{2}=\kappa M \pi_{\text {rel }}
$$

To produce the likelihood distributions shown in Figure 7 , we compute the likelihood by combining this equation and the measured values of $\theta_{\mathrm{E}}$ and $t_{\mathrm{E}}$ with the Galactic model (Han \& Gould 2003) assuming the distance to the GC is $8 \mathrm{kpc}$. Here systematic errors in parameters estimated in Section 4 are taken into account. This analysis yields that the primary is a K-dwarf with mass of $M_{l}=0.64_{-0.26}^{+0.21} M_{\odot}$ at $D_{l}=5.9_{-1.4}^{+0.9} \mathrm{kpc}$ and a planetary mass of $M_{p}=6.1_{-2.4}^{+2.0} \times 10^{-5} M_{\odot}=20_{-8}^{+7} M_{\oplus}$ and projected separation of $r_{\perp}=2.8_{-0.6}^{+0.5}$ AU. The physical

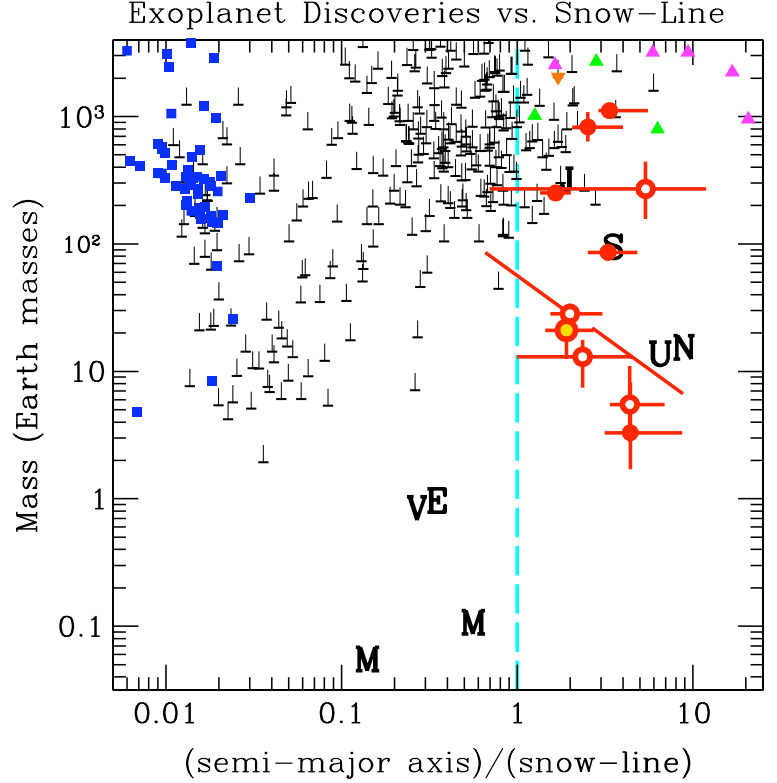

Figure 9. Known exoplanets as a function of mass vs. semimajor axis divided by the snow-line, which is taken to be at $a_{\text {snow }}=2.7 \mathrm{AU} M / M_{\odot}$. As in Figure 8, microlensing planets are indicated by red filled and open circles with error bars (see Section 7.2). OGLE-2007-BLG-368Lb is indicated by the gold-filled open circle. Blue dots represent the planets first detected by transits. The black bars with upward-pointing error bars are the planets detected via the radial velocity. The green and magenta triangles indicate the planets found by timing (including the pulsar planets) and by direct detection, respectively.

three-dimensional separation $a=3.3_{-0.8}^{+1.4} \mathrm{AU}$, can be estimated statistically by putting a planetary orbit at random inclination and phase (Gould \& Loeb 1992). The lens-source relative proper

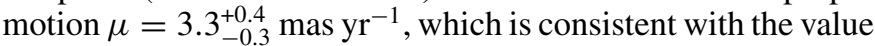
given by Equation (15), favors that the lens is in the bulge rather

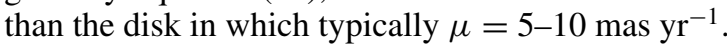

\subsection{Comparison to Other Known Exoplanets}

Figures 8 and 9 compare the masses and semimajor axes of the planets found by microlensing to those found by other methods. Figure 9 takes into account the different masses of the primary stars and uses the ratio of the semimajor axis to the position of the snow-line as the $x$-axis parameter in order to display the data in a way more relevant to planet formation theories.

The positions of all the microlensing planets on these plots are determined by a Bayesian analysis similar to the one we present for OGLE-2007-BLG-368Lb. However, there is a crucial distinction. The events plotted with red filled circle and error bars have masses determined either by microlensing parallax measurements or by direct identification of the lens star in Hubble Space Telescope (HST) images, so these can be considered to be actual measurements. The other microlensing planets, plotted with red open circle and error bars, are like OGLE-2007-BLG-368Lb, in that the light-curve measurements do not directly determine the lens system mass. For these events, the derived parameters have a significant dependence on the assumed prior, and we must be careful not to over-interpret the results. For example, we cannot use the results of these Bayesian analyses to study the probability that a star will host a planet as a function of its mass, because these estimates of the host star mass depend upon our prior assumptions about this planet hosting probability. Instead, such questions must be studied with a new Bayesian analysis using only directly measured quantities as constraints. 
There are planetary microlensing events that warrant some additional discussion. The Bayesian analyses for these events yield double-peaked likelihood functions. This gives results that are extremely sensitive to the prior assumptions, so one should not directly use the Bayesian results in these cases. Event MOA2007-BLG-400 has a severe $d \leftrightarrow 1 / d$ model degeneracy, which yields a factor of 10 uncertainty in the projected star-planet separation. We extend the error bars from the $1 \sigma$ lower limit on the semimajor axis from the $d<1$ solution to the $1 \sigma$ upper limit from the $d>1$ solution.

The other ambiguous planet is MOA-2008-BLG-310Lb (Janczak et al. 2010). This event is unusual because the kinematics favors a low-mass primary of $\sim 0.1 M_{\odot}$, while the excess flux seen in VLT/NACO images of the source star suggests a much more massive planetary host star with $M \sim 0.7 M_{\odot}$. But this excess flux could be due to a companion to the lens, source, or the chance superposition of an unrelated star. So the Bayesian analysis yields two peaks for the lens star (and planet) masses, but the relative weighting of these two peaks is quite sensitive to the assumed prior. So, as with MOA-2007-BLG-400, we use the $1 \sigma$ upper and lower limits on the high-mass and low-mass solutions for our error bars for this event. For the central point, we use the geometric mean of the peaks of the high-mass and low-mass solutions.

\section{CONSTRAINTS ON THE PLANETARY MASS FUNCTION}

In Figures 8 and 9, it appears that the distribution of planets found by microlensing is roughly independent of mass above $1 M_{\oplus}$, with perhaps a peak at $M \sim 10 M_{\oplus}$. However, the probability that a planet can be detected by microlensing depends on its mass, and these figures have not been corrected for the planet detection efficiency (Albrow et al. 2000; Gaudi \& Sackett 2000; Rhie et al. 2000). A full calculation of the planet detection efficiency (A. Cassan et al. 2010, in preparation) including detailed assessments of various potential systematics is beyond the scope of this paper, but we can obtain interesting constraints on the planetary mass function using a simple model for the relative planet detection efficiency.

For events with signals due to the planetary caustic (Gould \& Loeb 1992), there are some simple theoretical arguments regarding the dependence of the planet detection efficiency on the mass ratio, $q$. If we ignore finite source effects, which are usually unimportant for planets with masses $\gtrsim 10 M_{\oplus}$ (Bennett \& Rhie 1996), then the planetary caustic shape is nearly independent of $q$, and its area scales as $q$. We can define a planet detection region as the area of the lens magnification pattern that differs from the single-lens light curve (Paczyński 1986) by more than some threshold (either relative or absolute). With such a definition, the area of the planet detection region will scale as the size of the planetary caustic, as $q$. Then the probability that a given source trajectory will cross the planet detection region scales as the linear dimension of this region, which goes as $q^{1 / 2}$. So, in the limit of very good light curve coverage, the planet detection efficiency for planetary caustic events should scale as $q^{1 / 2}$. However, the duration of the planetary deviation also scales as $q^{1 / 2}$, and with sparse light curve coverage or large photometric error bars, the detection efficiency can scale as steeply as $q$. In general, we expect that situation to be in between these extremes for planetary caustic events, so that we should expect the planet detection efficiency to have some scaling intermediate between $q^{1 / 2}$ and $q$. We have calculated the detection efficiency for OGLE-2007-BLG-368 using the method of Rhie et al. (2000). Of course, it would be inconsistent to use the follow-up data that were taken because the planetary signal was recognized in such a calculation, so we have only included the regularly scheduled survey data in this calculation. The dependence of the detection efficiency on the detection threshold in different alert systems is negligible compared to the dependence on the light-curve coverage of the data set. This calculation gives a detection efficiency for OGLE-2007BLG-368 that scales as $\sim q^{0.8}$ at the range of $q$ appropriate for Neptune-mass to Jupiter-mass planets. This same scaling also holds true for the two other microlensing planets discovered through planetary caustic deviations, OGLE-2003-BLG-235 and OGLE-2005-BLG-390 (Kubas et al. 2008). For all the calculations in this section, we assume that the distribution of planets is uniform in $\log (d)$ for all separations, $d$, and we sum over all separations.

The situation is somewhat different for high magnification microlensing events, which are detected through perturbations of the central caustic. Since the linear size of the central caustic scales as $q$ (Dominik 1999), one might expect that the detection efficiency would scale more steeply than $q$ for the same reasons that the planetary caustic planet detection efficiency scales more steeply than $q^{1 / 2}$, but this is not the case. The reason for this is that for events of sufficiently high magnification, $A_{\max }>50$ or so, the planet detection efficiency for Jupitermass planets saturates at 1 for a wide range of separations. This is, in fact, the main reason why the observing groups focus on high magnification events (Griest \& Safizadeh 1998). The planet detection efficiency has been calculated for a number of high magnification events (Albrow et al. 2001; Gaudi et al. 2002; Rattenbury et al. 2002; Abe et al. 2004; Yoo et al. 2004b; Dong et al. 2006; Bennett et al. 2008; Nagaya 2009; Yee et al. 2009; Batista et al. 2009), and these events reveal detection efficiency scalings that range from $q^{0.7}$, for MOA-2006-BLG-130 and MOA-2007-BLG-192, to $q^{0.3}$ for OGLE-2008-BLG-279, which is the event with the highest planet detection sensitivity (Yee et al. 2009). Generally, the scaling is shallowest for the events with the highest sensitivity to planets and steeper for events with lower sensitivity due to lower peak magnification, less complete light-curve sampling, or less precise photometry. For the collection of high magnification events observed, we estimate that the mean detection efficiency scales as $q^{0.5 \pm 0.1}$, and for all microlensing events, we estimate that the detection efficiency scales as $q^{0.6 \pm 0.1}$.

We can now use the detection efficiency estimate to infer some properties of the distribution of planets in our Galaxy. In analogy to the stellar mass function, we define the planetary mass ratio function, $d N_{\mathrm{pl}} / d \log q$, such that the number of planets per star in a logarithmic mass ratio interval is given by $d N_{\mathrm{pl}} / d \log q$. We assume to have a power-law form for the planetary mass ratio function,

$$
\frac{d N_{\mathrm{pl}}}{d \log q}=N_{0} q^{n} \Theta\left(q-q_{0}\right) \Theta\left(q_{1}-q\right),
$$

where $q_{0}$ and $q_{1}$ are the lower and upper limits on the planetary mass ratio ( $q_{0}$ could alternatively be considered to be a lowmass-ratio cutoff on the planetary detection efficiency). Thus, $n=0$ would imply that there are an equal number of planets in every logarithmic mass interval, and $n=-1$ would imply that total mass of planets in every logarithmic mass interval is the same.

We can estimate the parameters, $N_{0}$ and $n$, that describe the planetary mass ratio function using a likelihood analysis. 


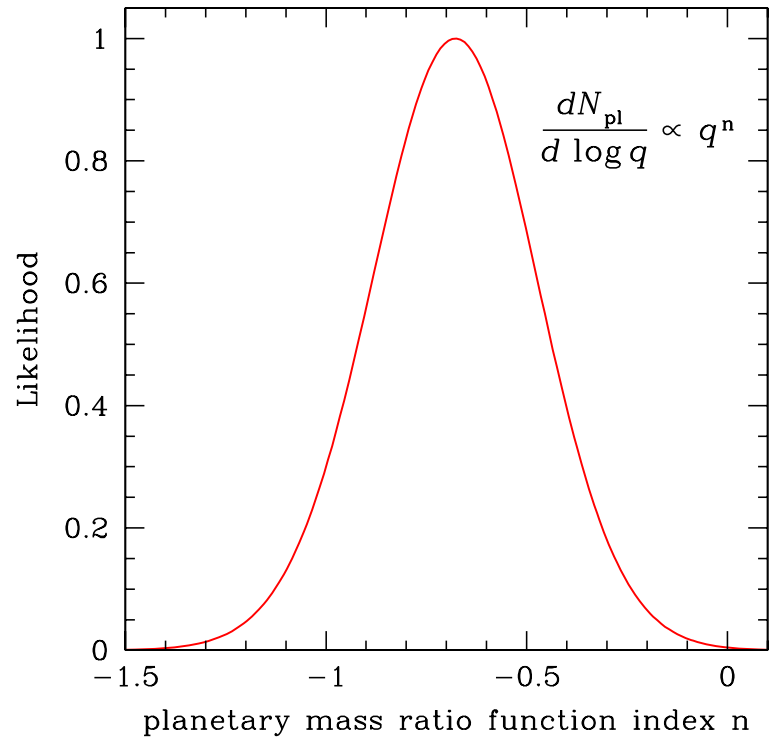

Figure 10. Probability distribution of the power law index, $n$, of the planetary mass ratio function, $\Psi(q)$, based upon the mass ratios of the 10 exoplanets detected by microlensing and our estimate of the planetary detection efficiency. This calculation yields $n=-0.68 \pm 0.20$, with a $95 \%$ confidence level upper limit of $n<-0.35$

(A color version of this figure is available in the online journal.)

The expression for the likelihood function for the planetary mass ratio function parameters is just the Poisson probability of finding the observed number of events, $N_{\text {obs }}$, times the product of the probability of finding events with each of the observed mass ratios, $q_{i}$. This can be written as

$$
\mathcal{L}\left(N_{0}, n\right)=e^{-N_{\exp }} \prod_{i}^{N_{\mathrm{obs}}} \frac{d N_{\mathrm{pl}}}{d \log q} \mathcal{E}\left(q_{i}\right),
$$

where $\mathcal{E}(q) \propto q^{0.6 \pm 0.1}$ is the planet detection efficiency and $N_{\text {exp }}$ is the number of events expected for the given $N_{0}$ and $n$ values (Alcock et al. 1996, 1997). However, since we have only calculated relative and not absolute efficiencies, we cannot calculate $N_{\exp }$ and we cannot hope to constrain $N_{0}$. Therefore, we adjust $\Phi_{0}$ so that $N_{\text {exp }}=N_{\text {obs }}$, and evaluate the likelihood function for only the power-law index, $n$, of the planetary mass ratio function. The resulting likelihood function based on the 10 planets discovered by microlensing is shown in Figure 10, and the resulting planetary mass ratio function index is $n=-0.68 \pm 0.20$, with a $95 \%$ confidence level upper limit of $n<-0.35$. The core of this distribution is similar to a Gaussian, but the distribution is skewed, with a higher probability of a $>2 \sigma$ deviation at small $n$ than at large $n$. This error bar includes the \pm 0.1 uncertainty in the detection efficiency power law index $\left(\mathcal{E}(q) \propto q^{0.6 \pm 0.1}\right)$. This result does have some dependence on our choice of the lower and upper cutoffs of $q_{0}=3 \times 10^{-5}$ and $q_{1}=0.015$, but the variation due to the choice of these cutoffs is much smaller than the resulting uncertainty in $n$.

This result for the power law index indicates that we should expect $7_{-3}^{+6}$ times as many cold Neptunes $\left(q \sim 5 \times 10^{-5}\right)$ as Jupiters $\left(q \sim 10^{-3}\right)$, with a $95 \%$ confidence level lower limit of 2.8 times as many cold Neptunes as Jupiters. This is in line with the basic predictions of the core accretion model (Ida \& Lin 2004; Laughlin et al. 2004), as these models predict a large population of Neptune-like, "failed Jupiter" cores to form beyond the snow-line, particularly for stars of less than a solar mass, which make up most of the sample probed by microlensing. However, it still may be possible to explain this result in the context of the gravitational instability theory (Boss 2006).

This power law index of $n=-0.68 \pm 0.20$ is steeper than (but consistent with) the index of $n=-0.31 \pm 0.20$ found by Cumming et al. (2008) for more massive planets orbiting mostly solar-type stars. This is also steeper than the mass function prediction of Mordasini et al. (2009) for solar-type stars, although this theoretical mass function is not a power law. Radial velocity surveys also find that hot Neptunes, with periods less than 50 days are quite common around $\mathrm{G}$ and $\mathrm{K}$ dwarfs (Mayor et al. 2009).

\section{DISCUSSION AND CONCLUSIONS}

We have presented the analysis of the OGLE-2007-BLG-368 planetary microlensing event, which indicates that the planet OGLE-2007-BLG-368Lb is a Neptune-mass planet. We also find evidence for low level systematic errors in the light curve, which however do not affect this conclusion. We estimate the systematic errors by taking the differences between the various models, i.e., the standard and xallarap with and without Kepler constraint.

By using a Bayesian analysis, we found the planet has a mass of $M_{p}=20_{-8}^{+7} M_{\oplus}$ and a projected separation of $r_{\perp}=$ $2.8_{-0.6}^{+0.5} \mathrm{AU}$ around a K-dwarf with mass of $M_{l}=0.64_{-0.26}^{+0.21} M_{\odot}$ at $D_{l}=5.9_{-1.4}^{+0.9} \mathrm{kpc}$. This is the fourth Neptune-mass planet detected by microlensing. In Figure 8 , we plot these planets as a function of mass versus semimajor axis along with all known exoplanets. Figure 9 is the same as Figure 8 but the semimajor axis is divided by the snow-line, which is taken to be at $a_{\text {snow }}=2.7 \mathrm{AU} M / M_{\odot}$. As for the microlensing planets in this figure, we are starting to see a broad concentration of $\sim 10 M_{\oplus}$ planets beyond the snow-line. This is as expected from the core accretion theory. This theory predicts that the most massive solid planetary cores should form beyond the snow-line, which then accrete nebular gas and become the gas giants around the solar-type star. On the other hand, they become Earth-mass to Neptune-mass icy rocky planets around M-dwarfs. Comparing four Neptune-mass, five Jovian planets and one between Neptune and Saturn found by microlensing, it confirms that cold Neptunes are relatively common around low-mass primary stars analyzed by Gould et al. (2006).

We have presented an analysis of the exoplanet mass ratio function. Assuming that the number of planets scales as a power law in the mass ratio, $q$, we define the mass ratio function as $d N_{\mathrm{pl}} / d \log q \propto q^{n}$ power law mass function, over the mass range of a few Jupiter masses down to a few Earth-masses, we find a power law index of $n=-0.68 \pm 0.20$, which indicates that Neptune-mass planets are substantially more common than Jupiter-mass planets.

The planetary signature of this event was detected in realtime in the data points from survey telescopes MOA and OGLE. Then the signature was greatly clarified by intensive follow-up observations prompted by the alert. This is a planetary caustic crossing event, the second of its kind after OGLE 2005-BLG390 (Beaulieu et al. 2006) among all planetary microlensing events. OGLE 2003-BLG-235 (Bond et al. 2004) crossed the planetary part of a resonant caustic. Although the time of the planetary deviation in these events cannot be predicted for planetary-caustic events, the potential event rate is higher than central-caustic event in which the time of the planetary deviation 
is known (Han \& Kim 2001). This discovery shows that the high cadence survey observations that MOA is conducting, have a great potential to increase the event rate of the planetary microlensing. In 2010, OGLE will upgrade its camera to $1.4 \mathrm{deg}^{2}$ FOV (OGLE-IV), which will enable OGLE to follow a similar strategy of high-cadence monitoring for planetary signals.

Multi-continent high-cadence observing will commence in 2010 with the start of the OGLE-IV project, and in future years will expand further when the Korean Microlensing Telescope Network (KMTNet) is commissioned. These improvements can be expected to dramatically increase the number of microlensing planets, and in particular those like OGLE-2007-BLG-368Lb, that are discovered via planetary-caustic perturbations.

This work is supported by the grant JSPS18253002 and JSPS20340052 (MOA). T.S. was supported by MEXT Japan, Grant-in-Aid for Young Scientists (B), 18749004 and Grant-inAid for Scientific Research on Priority Areas, "Development of Extra-solar Planetary Science,” 19015005. D.P.B. was supported by grants AST-0708890 from the NSF and NNX07AL71G from NASA. The OGLE project is partially supported by the Polish MNiSW grant N20303032/4275 to AU. Work by A.G. was supported by NSF grant AST-0757888. Work by B.S.G., A.G., and R.P. is supported by NASA grant NNG04GL51G Dave Warren provided financial support for Mt Canopus Observatory. C.H. was supported by Creative Research Initiative Program (2009-0081561) of National Research Foundation of Korea (CH). B.-G.P. and C.-U.L. were supported by the grant of Korea Astronomy and Space Science Institute.

\section{REFERENCES}

Abe, F., et al. 2004, Science, 305, 1264

Alard, C. 2000, A\&AS, 144, 363

Alard, C., \& Lupton, R. H. 1998, ApJ, 503, 325

Albrow, M. D., et al. 2000, ApJ, 535, 176

Albrow, M. D., et al. 2001, ApJ, 556, L113

Albrow, M., et al. 2009, MNRAS, 397, 2099

Alcock, C., et al. 1995, ApJ, 454, 125

Alcock, C., et al. 1996, ApJ, 461, 84

Alcock, C., et al. 1997, ApJ, 486, 697

Batista, V., et al. 2009, A\&A, 508, 467

Beaulieu, J.-P., et al. 2006, Nature, 439, 437

Bennett, D. P. 2009, ApJ, submitted (arXiv:0911.2703)

Bennett, D. P., \& Rhie, S. H. 1996, ApJ, 472, 660

Bennett, D. P., \& Rhie, S. H. 2002, ApJ, 574, 985

Bennett, D. P., et al. 2008, ApJ, 684, 663

Bennett, D. P., et al. 2009, ApJ, submitted (arXiv:0911.2706)

Bessell, M. S., \& Brett, J. M. 1988, PASP, 100, 1134

Bond, I. A., et al. 2001, MNRAS, 327, 868

Bond, I. A., et al. 2004, ApJ, 606, L155

Boss, A. P. 2006, ApJ, 644, L79

Bramich, D. M. 2008, MNRAS, 386, 77

Cardelli, J. A., Clayton, G. C., \& Mathis, J. S. 1989, ApJ, 345, 245

Claret, A. 2000, A\&A, 363, 1081

Cumming, A., Butler, R. P., Marcy, G. W., Vogt, S. S., Wright, J. T., \& Fischer, D. A. 2008, PASP, 120, 531

Dominik, M. 1999, A\&A, 349, 108
Dominik, M., et al. 2007, MNRAS, 380, 792

Dominik, M., et al. 2008, Astron. Nachr., 329, 248

Dong, S., et al. 2006, ApJ, 642, 842

Dong, S., et al. 2009a, ApJ, 695, 970

Dong, S., et al. 2009b, ApJ, 698, 1826

Gaudi, B. S., \& Gould, A. 1997, ApJ, 486, 85

Gaudi, B. S., \& Sackett, P. D. 2000, ApJ, 528, 56

Gaudi, B. S., et al. 2002, ApJ, 566, 463

Gaudi, B. S., et al. 2008, Science, 319, 927

Girardi, L., \& Salaris, M. 2001, MNRAS, 323, 109

Gould, A. 1992, ApJ, 392, 442

Gould, A., \& Loeb, A. 1992, ApJ, 396, 104

Gould, A., et al. 2006, ApJ, 644, L37

Gould, A., et al. 2009, ApJ, 698, L147

Griest, K., \& Hu, W. 1992, ApJ, 397, 362

Griest, K., \& Safizadeh, N. 1998, ApJ, 500, 37

Han, C., \& Gould, A. 1997, ApJ, 480, 196

Han, C., \& Gould, A. 2003, ApJ, 592, 172

Han, C., \& Kim, Y. 2001, ApJ, 546, 975

Ida, S., \& Lin, D. N. C. 2004, ApJ, 616, 567

Janczak, J., et al. 2010, arXiv:0908.0529

Kennedy, G. M., Kenyon, S. J., \& Bromley, B. C. 2006, ApJ, 650, L139

Kervella, P., \& Fouqué, P. 2008, A\&A, 491, 855

Kervella, P., Thévenin, F., Di Folco, E., \& Ségransan, D. 2004, A\&A, 426, 297

Konacki, M., et al. 2005, ApJ, 624, 372

Kubas, D., et al. 2008, A\&A, 483, 317

Lagrange, A. M., et al. 2009, A\&A, 493, L21

Laughlin, G., Bodenheimer, P., \& Adams, F. C. 2004, ApJ, 612, L73

Liebes, S. 1964, Phys. Rev., 133, 835

Mao, S., \& Paczyński, B. 1991, ApJ, 374, L37

Marcy, G. W., et al. 2005, ApJ, 619, 570

Marois, C., et al. 2008, Science, 322, 1348

Mayor, M., \& Queloz, D. 1995, Nature, 378, 355

Mayor, M., et al. 2004, A\&A, 415, 391

Mayor, M., et al. 2009, A\&A, 493, 639

Mordasini, C., Alibert, Y., Benz, W., \& Naef, D. 2009, A\&A, 501, 1161

Nagaya, M. 2009, Master's thesis, Nagoya Univ.

Nishiyama, S., et al. 2005, ApJ, 621, L105

Paczyński, B. 1986, ApJ, 304, 1

Paczyński, B., \& Stanek, K. Z. 1998, ApJ, 494, L219

Pravdo, S. H., \& Shaklan, S. B. 2009, ApJ, 700, 623

Rattenbury, N. J., Bond, I. A., Skuljan, J., \& Yock, P. C. M. 2002, MNRAS, 335,159

Reid, M. J. 1993, ARA\&A, 31, 345

Rhie, S. H., et al. 2000, ApJ, 533, 378

Salaris, M., \& Girardi, L. S. 2002, MNRAS, 337, 332

Schechter, P. L., Mateo, M., \& Saha, A. 1993, PASP, 105, 1342

Schmidt-Kaler, Th. 1982, in Landolt-Börnstein: Numerical Data and Functional Relationships in Science and Technology, Vol 2b, ed. K. Schaifers \& H. H. Voigt (Berlin: Springer)

Schneider, P., Ehlers, J., \& Falco, E. E. 1992, Gravitational Lenses (Berlin: Springer)

Smith, M. C., Mao, S., \& Woźniak, P. R. 2002, MNRAS, 332, 962

Stanek, K. Z., et al. 1997, ApJ, 477, 163

Sumi, T. 2004, MNRAS, 349, 193

Sumi, T., et al. 2003, ApJ, 591, 204

Tomany, A. B., \& Crotts, A. P. 1996, AJ, 112, 2872

Udalski, A. 2003, Acta Astron., 53, 291

Udalski, A., et al. 2004, Acta Astron., 54, 313

Udalski, A., et al. 2005, ApJ, 628, L109

Verde, L., et al. 2003, ApJS, 148, 195

Yee, J. C., et al. 2009, ApJ, 703, 2082

Yoo, J., et al. 2004a, ApJ, 603, 139

Yoo, J., et al. 2004b, ApJ, 616, 1204 